\title{
Tracing the evolution of the Galactic bulge with chemodynamical modelling of alpha-elements ${ }^{\star}$
}

\author{
A. C. S. Friaça and B. Barbuy \\ Universidade de São Paulo, IAG, Rua do Matão 1226, Cidade Universitária, 05508-900 São Paulo, Brazil \\ Received 21 October 2016 / Accepted 30 November 2016

\begin{abstract}
Context. Galactic bulge abundances can be best understood as indicators of bulge formation and nucleosynthesis processes by comparing them with chemo-dynamical evolution models.

Aims. The aim of this work is to study the abundances of alpha-elements in the Galactic bulge, including a revision of the oxygen abundance in a sample of 56 bulge red giants.

Methods. Literature abundances for $\mathrm{O}, \mathrm{Mg}, \mathrm{Si}, \mathrm{Ca}$ and $\mathrm{Ti}$ in Galactic bulge stars are compared with chemical evolution models. For oxygen in particular, we reanalysed high-resolution spectra obtained using FLAMES+UVES on the Very Large Telescope, now taking each star's carbon abundances, derived from $\mathrm{CI}$ and $\mathrm{C}_{2}$ lines, into account simultaneously.

Results. We present a chemical evolution model of alpha-element enrichment in a massive spheroid that represents a typical classical bulge evolution. The code includes multi-zone chemical evolution coupled with hydrodynamics of the gas. Comparisons between the model predictions and the abundance data suggest a typical bulge formation timescale of 1-2 Gyr. The main constraint on the bulge evolution is provided by the $\mathrm{O}$ data from analyses that have taken the $\mathrm{C}$ abundance and dissociative equilibrium into account. $\mathrm{Mg}, \mathrm{Si}, \mathrm{Ca}$ and Ti trends are well reproduced, whereas the level of overabundance critically depends on the adopted nucleosynthesis prescriptions.
\end{abstract}

Key words. Galaxy: bulge - stars: atmospheres - Galaxy: abundances - stars: abundances - Galaxy: stellar content

\section{Introduction}

Oxygen and magnesium are bona-fide alpha-elements produced uniquely in hydrostatic burning phases of massive stars, and their nucleosynthetic production is well-known. Oxygen in particular is among the most well-studied elements in stars, planetary nebulae and HII regions, and represents a link between stars and the interstellar medium. The elements $\mathrm{Si}, \mathrm{Ca}$ and $\mathrm{Ti}$ are instead mostly produced during explosive nucleosynthesis of massive stars (Woosley \& Weaver 1995, hereafter WW95).

In the Galactic bulge, the abundances of the alpha-elements $\mathrm{O}, \mathrm{Mg}, \mathrm{Si}, \mathrm{Ca}$ and $\mathrm{Ti}$ relative to iron are high, up to metallicities of $[\mathrm{Fe} / \mathrm{H}] \sim-0.6$, due to a high star-formation rate, meaning that the contribution from supernovae Type Ia only starts to increase the $\mathrm{Fe}$ abundance at these high metallicities. This behaviour is compatible with an intense star-formation rate in the Galactic bulge, which is assumed in most chemical evolution models (e.g. Matteucci \& Brocato 1990; Ballero et al. 2007; Grieco et al. 2012; Kobayashi \& Nakasato 2011; Cavichia et al. 2014).

In the present work, we present the predictions of a chemodynamical evolution model for the Galactic bulge, aiming at better undestanding the behaviour of alpha-element abundances as a function of metallicity. The code combines a multi-zone chemical evolution solver and a hydrodynamical code. In the model, a single massive dark halo hosts baryonic gas that can form stars. Feedback from the stellar population into the gas is included via several processes. Chemical enrichment and energy

\footnotetext{
* Observations collected both at the European Southern Observatory, Paranal, Chile (ESO programmes 71.B-0617A, 73.B0074A, and GTO 71.B-0196)
}

input by either supernovae ( $\mathrm{SNe}$ ) or planetary nebulae are assumed to occur at the end of stellar evolution. The input into the gas by the dying stars includes three contributions due to Type Ia $\mathrm{SNe}$ (SNe Ia), Type II SNe (SNe II) and quiescent stellar mass loss (Asymptotic giant branch stars, planetary nebulae and stellar winds), considering metallicity dependent yields. Then, the chemical evolution of the Galactic bulge is obtained, taking into account the gas flows in the system.

The alpha-elements $\mathrm{O}, \mathrm{Mg}, \mathrm{Si}, \mathrm{Ca}$ and $\mathrm{Ti}$ are investigated. In particular, we re-derive the oxygen abundances for the sample analysed by Zoccali et al. (2006), Lecureur et al. (2007) and Hill et al. (2011), now taking the carbon and nitrogen abundances into account, which is needed due to their influence in the molecular dissociative equilibrium. We also briefly discuss the comparison of oxygen abundances in the Galactic bulge and in thick disk stars.

In Sect. 2 the chemodynamical model is presented. In Sect. 3 the oxygen abundances of 56 bulge red giants are rederived. In Sect. 4, literature and present abundances in the Galactic bulge of $\mathrm{O}, \mathrm{Mg}, \mathrm{Si}, \mathrm{Ca}$ and $\mathrm{Ti}$ are compared with the chemodynamical models. Conclusions are then drawn in Sect. 5.

\section{Chemical evolution models of alpha-elements in the Galactic bulge}

Chemical evolution models for the Galactic bulge were presented by Matteucci \& Brocato (1990), Wyse \& Gilmore (1992), Samland \& Gerhard (2003), Ballero et al. (2007), Kobayashi \& Nakasato (2011), Grieco et al. (2012) and Cavichia et al. (2014), among others. 
The presently computed models assume a specific starformation rate (SFR), which is the inverse of the timescale for the system formation ( $v_{\mathrm{SF}}$, given in $\mathrm{Gyr}^{-1}$, is the ratio of the SFR in $M_{\odot} \mathrm{Gyr}^{-1}$ over the gas mass in $M_{\odot}$ available for star formation), of $v_{\mathrm{SF}}=0.5,1,2$ and $3 \mathrm{Gyr}^{-1}$. This means that $3 \mathrm{Gyr}^{-1}$ corresponds to a very fast timescale of $0.3 \mathrm{Gyr}$. A baryonic mass of $2 \times 10^{9} M_{\odot}$, and a dark halo mass $M_{\mathrm{H}}=1.3 \times 10^{10} M_{\odot}$ (total mass of $1.5 \times 10^{10} M_{\odot}$, thus reproducing the cosmological proportion of baryonic mass, $\Omega_{\mathrm{b}}=0.04$, to total matter mass, $\Omega_{\mathrm{m}}=0.3$ ) are assumed. In the present calculations, we adopt an $\Omega_{\mathrm{m}}=0.3, \Omega_{\Lambda}=0.7, H_{0}=70 \mathrm{~km} \mathrm{~s}^{-1} \mathrm{Mpc}^{-1}$ cosmology, with the corresponding age of the Universe being $13.799 \pm 0.038 \mathrm{Gyr}$ (Planck Collaboration I 2016).

The chemodynamical model is based on the model of galactic chemical evolution of Friaça \& Terlevich (1998), which combines a multi-zone chemical evolution solver and a hydrodynamical code. In the model, a single massive dark halo hosts baryonic gas that can form stars. The gas in turn receives feedback from the stellar population via heating, ionization, mechanical pressure and chemical enrichment. The stars are assumed to end either as supernovae $(\mathrm{SNe})$ or as planetary nebulae, when instantaneous ejection of mass and energy occurs. The energy input into the gas by the dying stars is divided into three contributions due to Type I SNe, Type II SNe and quiescent stellar mass-loss (AGBs/planetary nebulae and stellar winds).

The code allows spherical or spheroidal symmetry for the system, which is divided into cells and the hydrodynamical evolution of its interstellar medium (ISM) is calculated. The equations of chemical evolution for each cell are then solved taking into account the gas flow, and the evolution of the chemical abundances is obtained. For the chemical evolution calculations, a total of $\approx 300$ stellar generations are stored starting from the galaxy formation epoch. The model can be run in a one-dimensional (1D) mode (spherical symmetry), or in a two-dimensional (2D) mode if one wants to follow radial flows or polar outflows. In modelling the bulge, the code is run in the 1D mode.

It is assumed that the specific star-formation rate $v_{\mathrm{SF}}$ follows a power-law function of the gas density $v_{\mathrm{SF}} \propto \rho^{1 / 2}$. We included inhibition of star formation for expanding gas $(\nabla . u>0)$ or for too low densities, when the cooling is inefficient (that is, for a cooling time $t_{\text {coo }}=(3 / 2) k_{\mathrm{B}} T / \mu m_{\mathrm{H}} \Lambda(T) \rho$ longer than the dynamical time $\left.t_{\text {dyn }}=(3 \pi / 16 G \rho)^{1 / 2}\right)$ by multiplying $\nu_{\mathrm{SF}}$ by the inhibition factors $\left(1+t_{\text {dyn }} \max (0, \nabla . u)\right)^{-1}$ and $\left(1+t_{\text {coo }} / t_{\text {dyn }}\right)^{-1}$. The cooling function of the gas $\Lambda(T)$ is calculated consistently with the chemical abundances in the ISM, taking into account the abundances of the three main coolant elements, oxygen, carbon and iron separately. A Salpeter IMF is assumed, with $\mathrm{x}_{\mathrm{IMF}}=1.35$, in the mass range $0.1<M / M_{\odot}<100$.

The models use metallicity-dependent yields from SNe II, $\mathrm{SNe}$ Ia and intermediate-mass stars (IMS). Single stars with masses above $8 M_{\odot}$ are assumed to end their life as Type II SNe. The core-collapse SN II yields are adopted from WW95. However, for lower metallicities, we use the results of highexplosion-energy hypernovae (Umeda \& Nomoto 2002, 2003, 2005; Nomoto et al. 2006, 2013) because the latter models reproduce the large $[\mathrm{Zn} / \mathrm{Fe}]$ ratios of halo stars and lowmetallicity Damped Lyman- $\alpha$ systems (see Barbuy et al. 2015). At near-solar metallicities, the $\mathrm{Zn}$ underproduction problem (e.g. Timmes et al. 1995) can be solved by taking into account the effect of neutrinos on the electron excess $Y_{\mathrm{e}}$ of the innermost ejected zones (Fröhlich et al. 2006). Type Ia supernovae originate from binary systems of total mass in the range $3<m / M_{\odot}<$ 16 , in which the primary evolves until it becomes a C-O white dwarf. Subsequent mass-transfer from the evolved secondary triggers deflagration in the primary when the latter reaches the Chandrasekhar mass. The SNe Ia rates are calculated according to the prescriptions of Greggio \& Renzini (1983). A crucial parameter in this scenario is $A_{\mathrm{SNI}}$, the mass fraction of the IMF between $3<m / M_{\odot}<16$ that become binary systems giving rise to Type Ia SNe. Following Matteucci \& Tornambé (1987) and Matteucci (1992), $A_{\mathrm{SNI}}=0.1$ for most of the models. Yields of SNIa resulting from Chandrasekhar-mass white dwarfs, are taken from Iwamoto et al. (1999), namely their models W7 (progenitor star of initial metallicity $Z=Z_{\odot}$ ) and W70 (initial metallicity $Z=0)$. The yields for IMS $\left(0.8-8 M_{\odot}\right)$, with initial $Z=0.001,0.004,0.008,0.02$ and 0.4 , are from van den Hoek \& Groenewegen (1997) (variable $\eta_{\text {AGB }}$ case).

In order to gain insight into the formation and evolution of the Galactic bulge, in Sect. 4 the observational data are compared with the results of the present chemodynamical model describing a classical bulge.

\section{Re-derivation of the oxygen abundances}

The issue of the oxygen abundance in the Galactic bulge is not yet fully solved, partly due to technical approaches, that is, different lines are used in turn-off dwarfs or cool giants, and at different metallicities. In particular, the derivation of the oxygen abundance in giants depends on the carbon abundance, which is often difficult to obtain.

Essentially, four sets of lines can be used to derive oxygen abundances in old low-mass stars, noting that in most cases the different lines are not present in the same stars: (i) the forbidden $[\mathrm{OI}] \lambda 6300.31, \lambda 6363.79 \AA$ lines are measurable in giants of $[\mathrm{Fe} / \mathrm{H}]>-3.0$; they are reliable oxygen abundance indicators in cool giant stars, given that the lower level is the ground state and the upper level is controlled by collisions and therefore it is not affected by non-local thermodynamic equilibrium (NLTE) effects. Hydrodynamical three-dimensional (3D) model atmospheres show that this line is instead affected by granulation, increasing with decreasing metallicity and increasing temperatures, as shown by Nissen (2002), for example. Oxygen abundances in the Galactic bulge derived from these forbidden lines were presented by Zoccali et al. (2006), Lecureur et al. (2007), Fulbright et al. (2006, 2007), Alves-Brito et al. (2010) and Johnson et al. (2014). Bensby et al. (2004) also used these lines to examine a sample of thick disk giants; (ii) the permitted OI $\lambda 7771.96,7774.18$ and $7775.40 \AA$ triplet lines measurable in dwarfs and subgiants (in supergiants, also the weaker triplet at $\lambda$ 6156.03, 6156.80 and 6158.17 $\AA$ can be used); these lines are subject to strong NLTE effects. Recently, Steffen et al. (2015) were able to take into account the NLTE effects together with a 3D model atmosphere for the Sun. These lines were observed for microlensed bulge dwarfs by Bensby et al. (2013, and references therein); (iii) The infrared (IR) $\mathrm{X}^{2} \Pi$ vibration-rotation transitions of $\mathrm{OH}$ lines in the $H$ band, have measurable intensities down to $[\mathrm{Fe} / \mathrm{H}] \sim-3.0$ for giants with effective temperatures lower than $T_{\text {eff }} \sim 4300 \mathrm{~K}$. This line is present in both dwarf and giant stars, being stronger in dwarfs of the same temperature. Recently, Dobrovolkas et al. (2015) have shown that 3D calculations are needed to bring oxygen abundances from the IR OH lines into agreement with the forbidden atomic lines, in particular for metal-poor giants. These lines were analysed by Cunha \& Smith (2006), Meléndez et al. (2008), Ryde et al. (2010), Rich \& Origlia (2005) and Rich et al. (2012), and they are presently being extensively observed in bulge stars in the APOGEE survey (Majewski et al. 2016); (iv) the ultraviolet (UV) $\mathrm{OH}$ lines 
$\left(\mathrm{A}^{2} \Sigma-\mathrm{X}^{2} \Pi\right.$ electronic transition) are the only lines measurable in very metal-poor dwarfs. These lines were never observed in bulge stars due to the high extinction at these wavelengths.

The present sample of bulge stars consists of 56 red giants observed with the UVES spectrograph in FLAMES-UVES mode, at the Very Large Telescope at the European Southern Observatory, in Paranal, Chile. The stars were observed in four fields, which are the Baade's Window $\left(l=1.14^{\circ}, b=-4.2^{\circ}\right)$, a field at $\mathrm{b}=-6^{\circ}\left(l=0.2^{\circ}, b=-6^{\circ}\right)$, the Blanco field $\left(l=0^{\circ}\right.$, $\left.b=-12^{\circ}\right)$ and a field near NGC $6553\left(l=5.2^{\circ}, b=-3^{\circ}\right)$, as described in Lecureur et al. (2007) and Zoccali et al. (2006, 2008). Another 13 red clump stars in Baade's Window were observed and analysed by Hill et al. (2011) in GTO time.

The $\mathrm{C}, \mathrm{N}$ and $\mathrm{O}$ abundances of the 56 bulge giants were presented in Zoccali et al. (2006), Lecureur et al. (2007) and Hill et al. (2011). Despite having reported $\mathrm{C}$ and $\mathrm{N}$ abundances, the resulting oxygen abundances appear too high, as we explain in the present work: in Barbuy et al. (2015) the oxygen abundances from Zoccali et al. (2006) and Lecureur et al. (2007) were revised by reinspecting the $\mathrm{C}_{2}(0,1) 5635.5$ and $\mathrm{CN}(5,1)$ $6332.2 \AA$ lines, and a trend of lower oxygen abundances was found. The Barbuy et al. (2015) primary goal was to derive reliable $\mathrm{CN}$ line intensities in order to disentangle a blend of the Zn I $6362.35 \AA$ line from CN lines on its red wing. A fine-tuning of that work is reported here, additionally using the C I $5380.3 \AA$ line to verify the carbon abundances and derive more reliable oxygen abundances.

The difficulty of deriving reliable carbon abundances is due to the following reasons: (a) The carbon abundance derivation from optical spectra can be obtained from $\mathrm{CH}$ lines in the blue region, for example from the $G$-band at 4310-4315 $\AA$, however in the bulge the reddening in the blue becomes too high for faint stars due to the steep extinction law (e.g. Cardelli et al. 1989); (b) The $\mathrm{C}_{2}(0,1) 5635.2 \AA$ bandhead and atomic C I $5380.3 \AA$ lines are faint and in many stars they only allow an upper limit to be given.

\subsection{Calculations of $C, N$ and $O$ abundances}

The atmospheric models were obtained by interpolation in the grid of MARCS LTE models (Gustafsson et al. 2008). The spectrum synthesis code used is described in Barbuy et al. (2003) and Coelho et al. (2005). The stellar parameters reported in Table A.1 were adopted from the detailed analyses by Zoccali et al. (2006) and Lecureur et al. (2007) for 43 bulge field red giants. An additional 13 field red clump stars were analysed by Hill et al. (2011) based on both the UVES and the GIRAFFE spectra. We adopted the stellar parameters derived from the UVES spectra, which were not given in Hill et al. (2011) but already reported in Barbuy et al. (2013).

We note that in Barbuy et al. (2015) we revised the CNO abundances, with the exclusive aim of reproducing well the $\mathrm{CN}$ lines blending the $\mathrm{ZnI} 6362.35 \AA$ line. We used the $\mathrm{CN}$ lines from the laboratory measurements by Davis \& Phillips (1963). We initially adopted the $\mathrm{C}, \mathrm{N}$ and $\mathrm{O}$ abundances derived in Lecureur et al. (2007). Based on the observed profile of the $\mathrm{Zn}$ I $6362.35 \AA$ line, however, we realised that in some cases the $\mathrm{CN}$ blend was overestimated, producing a spurious asymmetry in the red wing of the Zn I $6362.35 \AA$ line. We then recomputed C, N and $\mathrm{O}$ for all sample stars, given the influence of the $\mathrm{CN}$ blend in the $\mathrm{Zn}$ abundance derivation from the Zn I $6362.35 \AA$ line. Oxygen was also derived for the two most metal-poor stars of
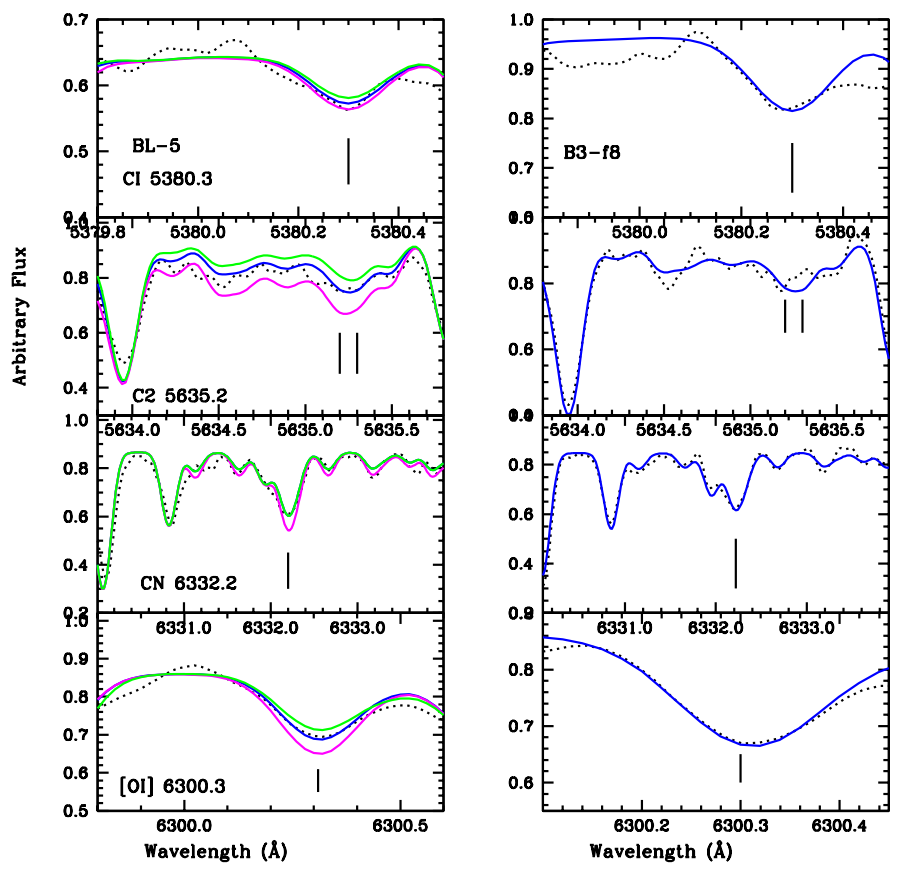

Fig. 1. Fits to the features CI 5380.3, C $25635.2, \mathrm{CN} 6332.2$ and [OI] $6300.3 \AA$ lines and bandheads, with the abundances reported in Table A.1, for the stars BL-5 and B3-f8. For BL-5 calculations with \pm 0.1 (green for minus, magenta for plus) for $\mathrm{C}, \mathrm{N}$ and $\mathrm{O}$ are shown.

the sample, BW-f4 and BW-f8, for which no previous derivation was available.

In the present work the derivation of $\mathrm{C}, \mathrm{N}$ and $\mathrm{O}$ abundances was carried out by also fitting the C I $5380.3 \AA$ line, together with the Swan $C_{2}(0,1) A^{3} \Pi-X^{3} \Pi$ bandhead at 5635.2 $\AA$, the red $\mathrm{CN}(5,1) \mathrm{A}^{2} \Pi-\mathrm{X}^{2} \Sigma$ bandhead at $6332.18 \AA$ and the forbidden oxygen [OI]6300.311 $\AA$ line. For the [OI] $6300.311 \AA$ line, a $\log g f=-9.716$ was used, and blends with Ni I 6300.330 and $6300.350 \AA$ lines, with $\log g f=-2.275$ and -2.695 , are adopted. For the C I $5380.337 \AA$ line, we use $\log g f=-1.72$, intermediate between the values of -1.616 and -1.842 given in NIST and VALD databases, respectively.

Fits to these $\mathrm{CNO}$ abundance indicators are illustrated in Fig. 1 for stars B3-f8 and BL-5. For BL-5, the changes due to $\Delta \mathrm{C}, \mathrm{N}, \mathrm{O} \pm 0.1$ are shown. These features have to be fitted iteratively, given that a change in the abundance of any of them has an impact on the molecular dissociative equilibrium. For example, a change in oxygen abundance will have impact on the $\mathrm{C}_{2}$ and $\mathrm{CN}$ lines.

In Table A. 1 the original $\mathrm{C}, \mathrm{N}$ and $\mathrm{O}$ abundances from Lecureur et al. (2007), the preliminary revision reported in Barbuy et al. (2015) and the presently fully revised C, N and $\mathrm{O}$ abundances for the 56 sample red giants are given.

\subsection{Uncertainties}

We have adopted uncertainties in the atmospheric parameters of $\pm 150 \mathrm{~K}$ for effective temperature, \pm 0.20 for surface gravity, \pm 0.10 in $[\mathrm{Fe} / \mathrm{H}]$ and $\pm 0.10 \mathrm{~km} \mathrm{~s}^{-1}$ for microturbulent velocity, as explained in Barbuy et al. (2013).

The errors in the abundance ratios $[\mathrm{O} / \mathrm{Fe}]$ were computed by fitting the $\mathrm{CI}, \mathrm{C}_{2}, \mathrm{CN}$ and [OI] $6300.311 \AA$ lines with the modified atmospheric model. The reported error corresponds to the difference needed to return from the value with the modified 
Table 1. Abundance uncertainties for star BL-5.

\begin{tabular}{lccccc}
\hline \hline Abundance & $\Delta T$ & $\Delta \log g$ & $\Delta v_{t}$ & {$[\mathrm{C} / \mathrm{Fe}]$} & $\left(\sum x^{2}\right)^{1 / 2}$ \\
& $150 \mathrm{~K}$ & $0.2 \mathrm{dex}$ & $0.1 \mathrm{~km} \mathrm{~s}^{-1}$ & & \\
$(1)$ & $(2)$ & $(3)$ & $(4)$ & $(5)$ & \\
\hline$[\mathrm{C} / \mathrm{Fe}](\mathrm{CI})$ & -0.05 & -0.001 & +0.00 & - & 0.05 \\
{$[\mathrm{C} / \mathrm{Fe}]\left(\mathrm{C}_{2}\right)$} & -0.005 & +0.005 & +0.00 & - & 0.07 \\
{$[\mathrm{~N} / \mathrm{Fe}]$} & +0.005 & +0.01 & +0.00 & \pm 0.15 & 0.16 \\
{$[\mathrm{O} / \mathrm{Fe}]$} & -0.01 & -0.08 & +0.00 & \pm 0.005 & 0.08 \\
\hline
\end{tabular}

Notes. $\Delta T_{\text {eff }}=+150 \mathrm{~K}, \Delta \log g=+0.2, \Delta v_{\mathrm{t}}=0.1 \mathrm{~km} \mathrm{~s}^{-1}$, and $\Delta[\mathrm{C} / \mathrm{Fe}]= \pm 0.1$, and the corresponding total error. The errors correspond to the difference needed to return to the adopted results.

model atmosphere to the adopted one. The error in the element abundance ratios, induced by a change of $\Delta T_{\text {eff }}=+150 \mathrm{~K}$, $\Delta \log g=+0.2$ and $\Delta v_{\mathrm{t}}=0.1 \mathrm{~km} \mathrm{~s}^{-1}$ are given. We also add the error induced by a variation in the carbon abundances of $\Delta[\mathrm{C} / \mathrm{Fe}]= \pm 0.1$ and computed the error of a metallicity variation of $\Delta[\mathrm{Fe} / \mathrm{H}]=-0.1$; the effect of the latter on abundance ratios is negligible. These errors and their quadratic sums are given in Table 1 for the star BL-5, noting that the total error is overestimated because the stellar parameters are covariant.

\subsection{Comparison with literature oxygen abundances}

In Table 2 we report the stellar parameters and oxygen abundances by Ryde et al. (2010), where some stars of the present sample were observed in the infrared. There is good agreement between the results from the two analyses for the same stars.

In Fig. 2 we show $[\mathrm{O} / \mathrm{Fe}]$ vs. $[\mathrm{Fe} / \mathrm{H}]$ in the metallicity range $-1.5<[\mathrm{Fe} / \mathrm{H}]<+0.7$, with the present results compared to literature results for the bulge (upper panel), and for thick disk stars (lower panel). The bulge samples are the bulge dwarfs by Bensby et al. (2013), giant stars from Alves-Brito et al. (2010) that were carried out in the optical for the same stars as in Meléndez et al. (2008), Cunha \& Smith (2006), Ryde et al. (2010), Rich et al. (2012), Johnson et al. (2014), Rich \& Origlia (2005) and Fulbright et al. (2006, 2007).

In the lower panel, the present oxygen abundances for 56 bulge giants are plotted together with thick disc stars analysed by Alves-Brito et al. (2010), Bensby et al. (2004) and Reddy et al. (2006).

In Table 3 we have gathered the solar oxygen and iron abundances used by these different authors, and have applied a correction to all of them, to be compatible with the value given by Steffen et al. (2015) of $A(\mathrm{O})=8.76$ and $A(\mathrm{Fe})=7.50$. The reasoning is that if a higher $A(\mathrm{O})$ value is assumed for the Sun, a lower value for the star will be given, therefore the correction has to be added to the oxygen abundances reported by the authors.

In Table 3 we also include the $A(\mathrm{O})$ and $A(\mathrm{Fe})$ solar abundances according to Allende-Prieto et al. (2001), Asplund et al. (2009), Grevesse \& Sauval (1998), Lodders (2009), Kurucz (1993), and Steffen et al. (2015).

The presently revised oxygen abundances in bulge stars are in very good agreement with those by Cunha \& Smith (2006) and Ryde et al. (2010), who used $H$-band $\mathrm{OH}$ lines to derive the oxygen abundances, taking into account the molecular dissociative equilibrium, and simultaneously deriving the carbon and nitrogen abundances. It appears to us that this is the reason for the agreement, and the reliability of these results. Rich et al. (2012) and Rich \& Origlia (2005) also used infrared lines, and should have taken into account the $\mathrm{C}$ and $\mathrm{N}$ abundances, but the $\mathrm{C}$ and $\mathrm{N}$ abundances are not reported, and consequently their method is not clear. Bensby et al. (2013, and references therein) derived abundances for microlensed bulge dwarfs. They used the permitted 7774-7 $\AA$ oxygen triplet lines, which are strongly subject to NLTE effects (Kiselman 2001; Steffen et al. 2015). The higher effective temperatures of turn-off dwarfs imply that there is no significant molecule formation, and in this case, it is the NLTE effects that cause a tendency to yield higher oxygen abundances.

Fulbright et al. (2006, 2007), Alves-Brito et al. (2010) and Johnson et al. (2014) used the reliable line of [OI]6300 $⿱$, but without taking the $\mathrm{C}$ and $\mathrm{N}$ abundances into consideration.

For these reasons, it appears that the present results, together with those by Ryde et al. (2010) and Cunha \& Smith (2006) are, in principle, the correct ones.

The abundance results for 264 RGB giants in three off-axis fields $(1, \mathrm{~b})=(-5.5,-7),(-4,-9)$ and $(+8.5,+9)$ from Johnson et al. (2013) were not included as although these authors took into account the $\mathrm{C}$ and $\mathrm{N}$ abundances very carefully, there is no mention of verification of blending with telluric lines. From some of their very high values of $[\mathrm{O} / \mathrm{Fe}]$ it seems that there was contamination in a number of stars. Besides, and maybe partly due to this effect, the results show a large spread, and for this reason they were not considered here. Their $\mathrm{Si}$ and $\mathrm{Ca}$ abundances also show some spread, probably due to their use of low resolution $(R \sim 18000)$ spectra. On the other hand, their work provides important confirmation of similar mean $[\mathrm{O} / \mathrm{Fe}],[\mathrm{Si} / \mathrm{Fe}],[\mathrm{Ca} / \mathrm{Fe}]$ vs. $[\mathrm{Fe} / \mathrm{H}]$ in different locations in the bulge.

\section{Chemical evolution models compared with alpha-element abundances}

Oxygen: Figs. 3 and 4 compare the $[\mathrm{O} / \mathrm{Fe}]$ vs. $[\mathrm{Fe} / \mathrm{H}]$ data from the presently rederived oxygen abundances, and literature data for bulge giants from Cunha \& Smith (2006) and Ryde et al. (2010), which we selected as being the most reliable results, to the predictions of the chemodynamical models for a classical bulge. Figures $3 a$ and $b$, exhibits the results of the model with specific star-formation rates of $v_{\mathrm{SF}}=3 \mathrm{Gyr}^{-1}$, with and without hypernovae. In the metallicity range considered, the effect of hypernovae is negligible, as can be verified in Fig. 3 .

The comparison between the predictions of the models including hypernovae with those without them, shows that the nucleosynthesis prescriptions are not critical for the $[\mathrm{O} / \mathrm{Fe}]$ ratios. In other words, the trend of $[\mathrm{O} / \mathrm{Fe}]$ vs. $[\mathrm{Fe} / \mathrm{H}]$ is a very robust indicator on the process of bulge formation. For this reason, despite the difficulties in obtaining oxygen abundances in bulge stars (for example due to NLTE effects on the permitted lines, or the need to take into account the carbon abundance when using the forbidden [OI]6300.3 $\AA$ line in cool stars), this effort is valuable because they are a prime probe for bulge formation.

Figures $4 \mathrm{a}$ and $\mathrm{b}$ shows the model predictions for $v_{\mathrm{SF}}=$ $1 \mathrm{Gyr}^{-1}$ and $v_{\mathrm{SF}}=0.5 \mathrm{Gyr}^{-1}$. It appears that the specific starformation rate in the range $v_{\mathrm{SF}}=0.5-1 \mathrm{Gyr}^{-1}$ is appropriate for describing the oxygen abundances from the present work, together with the oxygen abundances from Cunha \& Smith (2006) and Ryde et al. (2010). Figure 4c shows the same model as in panel a, but with a top-heavy IMF $\left(x_{\mathrm{IMF}}=1.05\right)$. This fit shows that a top-heavy IMF is not suitable.

Oxygen in bulge vs. thick disk stars: Fig. 3c compares the same model as in panel a with the data for the thick disk dwarfs from Bensby et al. (2004) and thick disk giants from Alves-Brito et al. (2010), together with the present oxygen abundances for 56 bulge giants. It should be noted that a thick disk is formed by a series of processes that are not included in the present 
A. C. S. Friaça and B. Barbuy: Chemodynamical evolution model of alpha-elements in the Galactic bulge

Table 2. Stellar parameters and CNO abundances from Ryde et al. (2010), Zoccali et al. (2006, 2008), and Barbuy et al. (2015).

\begin{tabular}{lccrr}
\hline \hline Star & $\begin{array}{l}T_{\text {eff }}, \log g,[\mathrm{Fe} / \mathrm{H}], v_{t} \\
\text { Ryde }+10\end{array}$ [C, N, O/Fe] & $\begin{array}{r}T_{\text {eff }}, \log g,[\mathrm{Fe} / \mathrm{H}], v_{t} \\
\text { Zoccali }+08, \text { Barbuy }+15\end{array}$ \\
\hline B3-b1 & $4365 / 2.0 /-0.73 / 1.5$ & $0.090,0.030,0.39$ & $4300 / 1.7 /-0.78 / 1.5$ & $0.1,0.6,0.4$ \\
B3-b8 & $4250 / 1.5 /-0.69 / 1.4$ & $-0.10,0.210 .39$ & $4400 / 1.8 /-0.62 / 1.4$ & $-0.16,0.3,0.25$ \\
BW-b6 & $4340 / 2.2 /-0.16 / 1.5$ & $0.13,0.27,0.43$ & $4200 / 1.7 /-0.25 / 1.3$ & $-0.3,0.9,0.25$ \\
BW-f6 & $4150 / 1.5 /-0.31 / 1.6$ & $0.050,0.24,0.28$ & $4100 / 1.7 /-0.21 / 1.5$ & $0.08,0.4,0.18$ \\
B6-f1 & $4030 / 1.3 /-0.08 / 1.5$ & $-0.10,0.37,0.030$ & $4200 / 1.6 /-0.01 / 1 / 5$ & $0.0,0.35,0.07$ \\
B6-b8 & $3985 / 1.1 /-0.14 / 1.3$ & $-0.050,0.44,0.10$ & $4100 / 1.6 / 0.03 / 1.3$ & $0.0,005,0.0$ \\
B6-f7 & $4315 / 1.9 /-0.34 / 1.6$ & $0.14,0.36,0.57$ & $4300 / 1.7 /-0.42 / 1.6$ & $0,0.3,0.1$ \\
Arp4203 & $3815 / 0.35 /-1.25 / 1.8$ & $-0.66,1.03,0.040$ & - & - \\
Arp4329 & $4153 / 1.15 /-1.02 / 1.5$ & $-0.11,0.28,0.41$ & - & - \\
Arp1322 & $4250 / 1.5 /-0.16 / 1.5$ & $-0.090,0.38,0.24$ & - & - \\
\hline
\end{tabular}
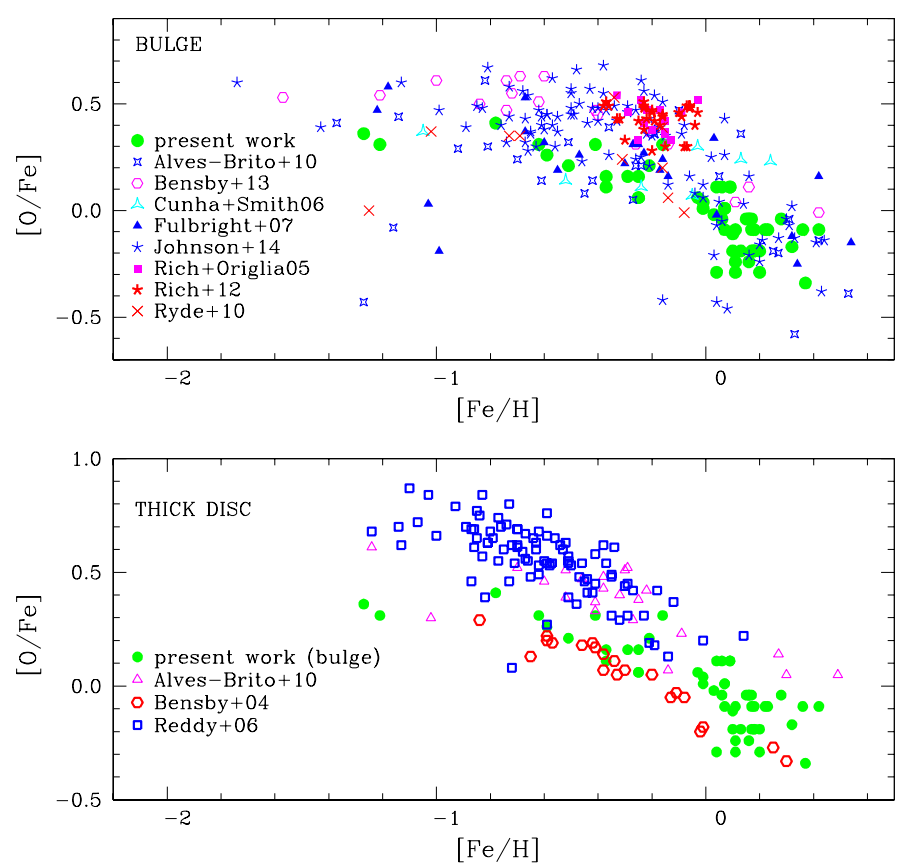

Fig. 2. $[\mathrm{O} / \mathrm{Fe}]$ vs. $[\mathrm{Fe} / \mathrm{H}]$ for present results compared with published abundances for halo and bulge stars. Panel a): plotted in the metallicity range $-1.5<[\mathrm{Fe} / \mathrm{H}]<+0.7$, corresponding to that of sample bulge giant stars, compared with the bulge dwarfs by Bensby et al. (2013), giant stars from Alves-Brito et al. (2010) which is a revision of Meléndez et al. (2008), Cunha \& Smith (2006), Johnson et al. (2014), Rich \& Origlia (2005) and Fulbright et al. (2006, 2007); panel b): bulge giants from the present work, and thick disk stars by Alves-Brito et al. (2010), Bensby et al. (2004) and Reddy et al. (2006).

models. This comparison is intended to verify published conclusions on the bulge and thick disk stars which give the same results (Meléndez et al. 2008; Alves-Brito et al. 2010; Gonzalez et al. 2011), among others. In all cases the oxygen indicator is the forbidden [OI]6300 $\AA$ line. The data are overplotted with the present chemo-dynamical models as in Fig. 3a. In the metallicity range of thick disk stars $(-1.2 \lesssim[\mathrm{Fe} / \mathrm{H}] \lesssim 0.0)$ there is very good agreement between the present results on oxygen abundances for the bulge giants and thick disk stars by Bensby et al. (2004). Whereas the oxygen abundances from Bensby et al. (2004) are remarkably similar to the present bulge giants, the bulge thick disk giants by Alves-Brito et al. (2010) show higher
Table 3. A. Oxygen solar abundances from the literature.

\begin{tabular}{lcll}
\hline \hline A. Sun & $A(\mathrm{O})_{\odot}$ & \multicolumn{1}{c}{$A(\mathrm{Fe})_{\odot}$} & Model \\
\hline Kurucz93 & 8.89 & 7.63 & $1 \mathrm{D}$ \\
Grevesse+98 & 8.87 & 7.50 & $1 \mathrm{D}$ \\
Allende-Prieto+01 & 8.69 & - & $3 \mathrm{D}$ \\
Allende-Prieto+01 & 8.77 & - & $1 \mathrm{D}$ \\
Asplund+09 & 8.69 & 7.50 & $3 \mathrm{D}$ \\
Lodders09 & 8.73 & 7.45 & - \\
Steffen+15 & 8.76 & - & $3 \mathrm{D}$ \\
\hline B. Bulge & $A(\mathrm{O}) \odot$ & $A(\mathrm{Fe}) \odot$ & Correction \\
\hline Rich+Origlia05 & 8.87 & 7.50 & +0.11 \\
Cunha+Smith06 & 8.77 & 7.50 & +0.01 \\
Fulbright+07 & 8.69 & 7.50 & -0.08 \\
Melendez+08 & 8.72 & 7.48 & -0.02 \\
Alves-Brito+10 & 8.74 & 7.53 & -0.04 \\
Reddy+06 & 8.73 & 7.50 & -0.03 \\
Ryde+10 & 8.72 & 7.50 & -0.04 \\
Rich+12 & 8.87 & 7.50 & +0.11 \\
Johnson+14 & 8.69 & 7.50 & -0.08 \\
Bensby+04 & 8.71 & 7.56 & -0.11 \\
Bensby+13 & 8.85 & 7.56 & +0.03 \\
present & 8.77 & 7.50 & +0.01 \\
\hline
\end{tabular}

Notes. B. Oxygen solar abundance adopted in studies of bulge star samples.

abundances and do not fit the present results for bulge giants. Note that Alves-Brito et al. did not take into account the $\mathrm{C}$ and $\mathrm{N}$ abundances, and their results may be overestimates. The same is true for oxygen abundances in thick disk stars by Reddy et al. (2006), where the permitted OI 7774-7 $\AA$ lines were analysed.

A further inspection of published available data is given in Fig. 5, showing $[\mathrm{O} / \mathrm{Fe}]$ vs. $[\mathrm{Fe} / \mathrm{H}]$ for the present results for bulge giants compared to thick disk and bulge giants by Alves-Brito et al. (2010), and thick disk and bulge dwarfs by Bensby et al. $(2004,2013)$. We preferred to use only the sample and results from Bensby et al. (2004) for 73 dwarf $F$ and $G$ thick disk 


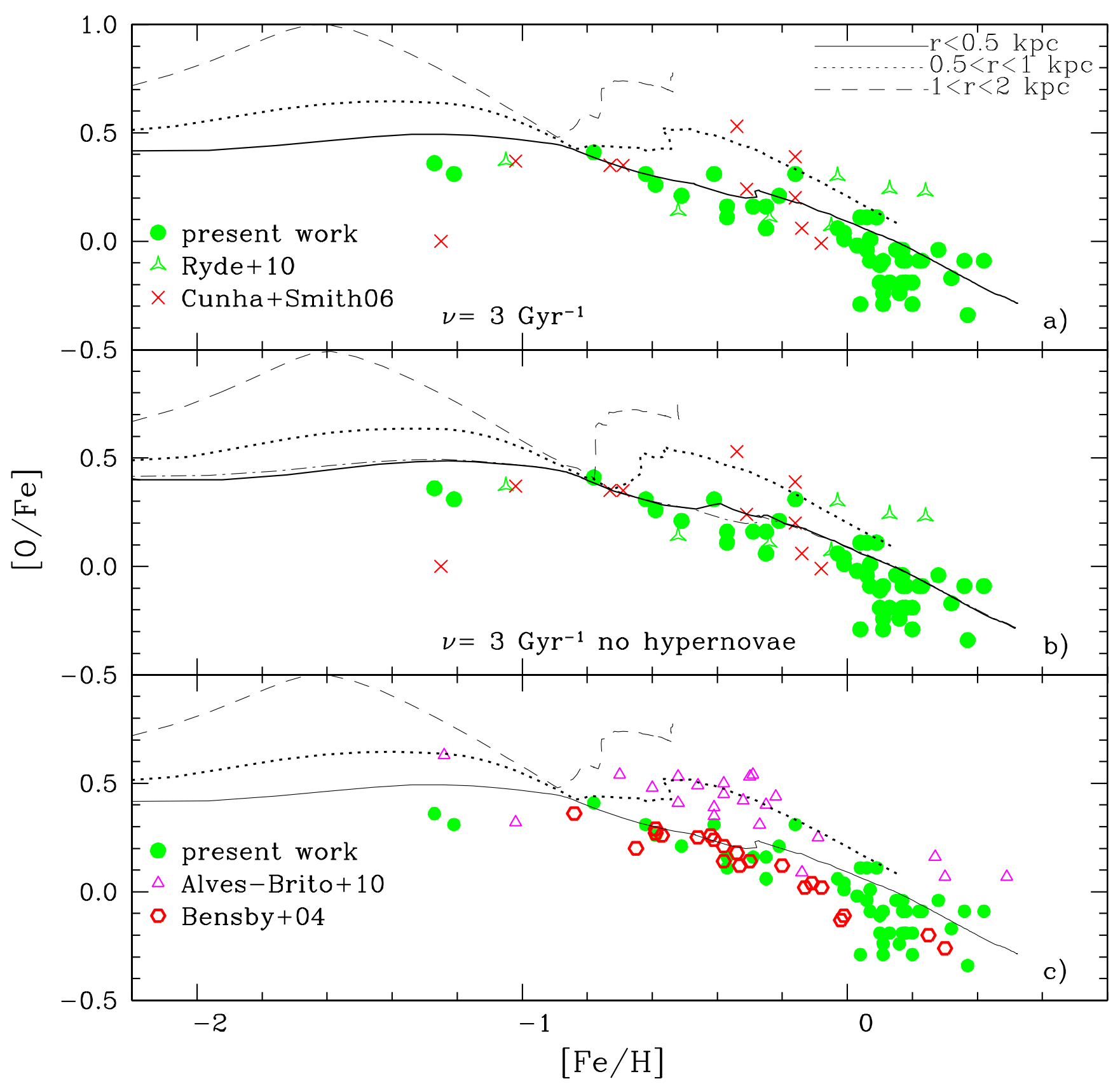

Fig. 3. $[\mathrm{O} / \mathrm{Fe}]$ vs. $[\mathrm{Fe} / \mathrm{H}]$ for the present results together with literature abundances for and bulge and thick disk stars and the predictions of the chemodynamical model for the bulge. Panel a): the data derived for the sample bulge giant stars are compared with the results for bulge giants from Cunha \& Smith (2006) and Ryde et al. (2010). The lines are the predictions of the model assumig a specific star formation rate of $v_{\mathrm{SF}}=3 \mathrm{Gyr}^{-1}$. Panel b): same data as in panel a) and the predicted $\mathrm{O} / \mathrm{Fe}]$ vs. $[\mathrm{Fe} / \mathrm{H}]$ for the model with no hypernovae. The dot-dashed line is the prediction by the model with hypernovae for $r<0.5 \mathrm{kpc}$; panel c): the $[\mathrm{O} / \mathrm{Fe}]$ vs. $[\mathrm{Fe} / \mathrm{H}]$ of the thick disk dwarfs from Bensby et al. (2004), thick disk giants from Alves-Brito et al. (2010) are superposed on the model predictions of panel a).

stars where the more reliable forbidden [OI] $6300.3 \AA$ line was employed and did not use the Bensby et al. (2014) data for thick disk stars given the dispersion in the oxygen abundance.

The present bulge giants and the bulge giants from Alves-Brito et al. (2010) show very good agreement. Despite the claim by Alves-Brito et al. (2010) that their bulge and thick disk giants show similar behaviour, a covariance analysis comparing the linear regressions of the two groups indicates that the thick disk giants have oxygen abundances higher than those of the bulge giants. A t-test applied to their data with $[\mathrm{Fe} / \mathrm{H}] \geq-1.0$ allows for rejection of the null hypothesis that both subsamples are drawn from the same-population at a $0.03 \%$ significance level, providing an upward average shift of 0.20 for the $[\mathrm{O} / \mathrm{Fe}]$ values of thick disk data with respect to the bulge data. Furthermore, the present bulge giants and the thick disk dwarfs by Bensby et al. (2004) show excellent agreement. Nevertheless, the bulge dwarfs by Bensby et al. (2013) are clearly enhanced with respect to the present bulge giants and thick disk dwarfs of Bensby et al. (2004). In this case, the covariance analysis rejects the hypothesis that both subsamples are drawn from the same-population at a $<0.01 \%$ significance level, but providing a downward average shift of 0.30 for the $[\mathrm{O} / \mathrm{Fe}]$ values of thick disk data with 
A. C. S. Friaça and B. Barbuy: Chemodynamical evolution model of alpha-elements in the Galactic bulge

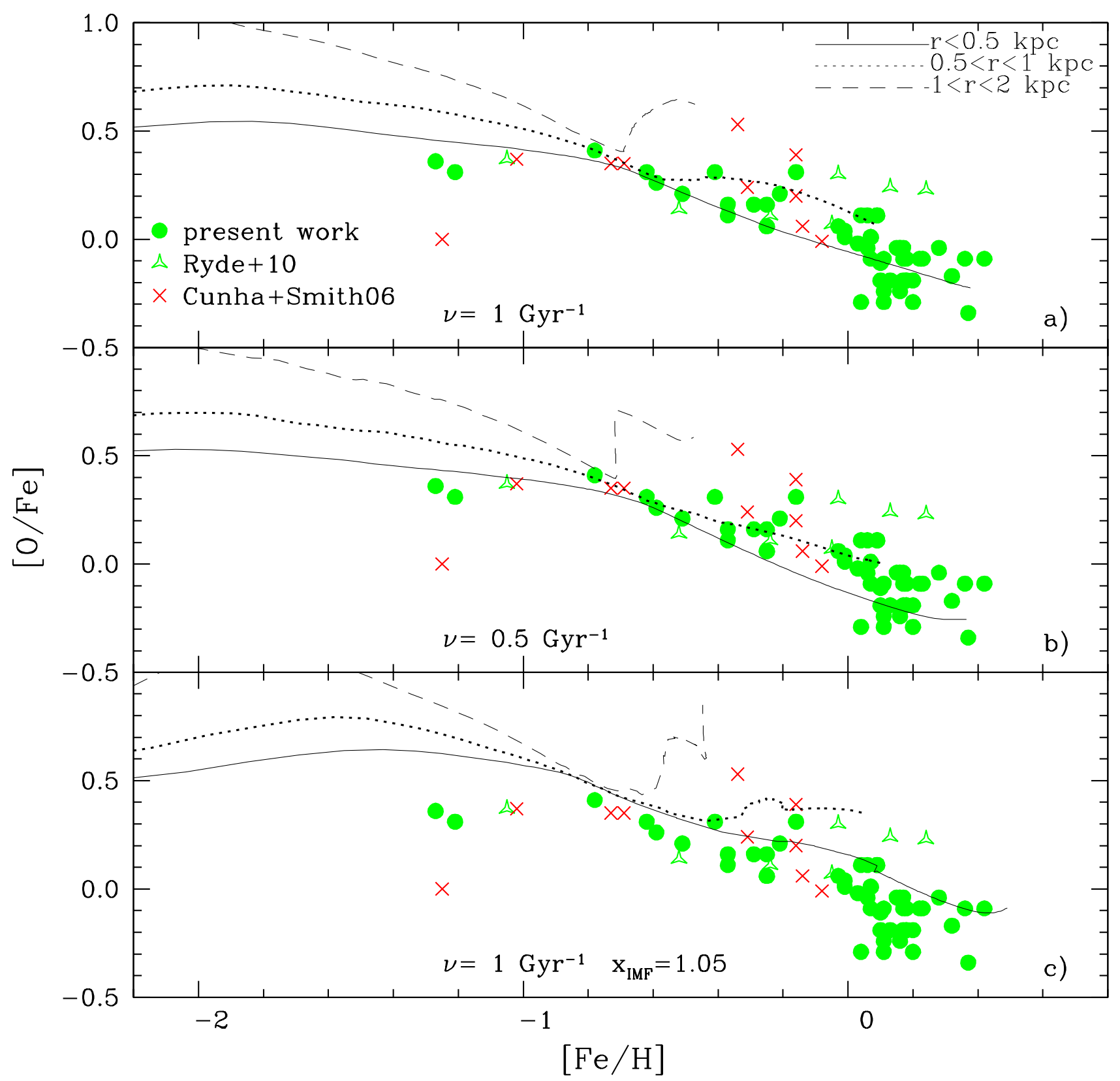

Fig. 4. $[\mathrm{O} / \mathrm{Fe}]$ vs. $[\mathrm{Fe} / \mathrm{H}]$ for the present results together with the results for bulge giants from Cunha \& Smith (2006) and Ryde et al. (2010) are compared to the predictions of the chemodynamical model for the bulge. Panel a): results from the model with $v_{\mathrm{SF}}=1 \mathrm{Gyr}^{-1}$. Panel b): results from the model with $v_{\mathrm{SF}}=0.5 \mathrm{Gyr}^{-1}$. Panel c): the same model as in panel a), but with a top-heavy IMF $\left(x_{\mathrm{IMF}}=1.05\right)$.

respect to the bulge data. These results illustrate why this discussion is inconclusive: it is not clear if the thick disk would be more oxygen-rich than bulge stars as hinted by results from Alves-Brito et al. (2010), or if the bulge stars would be more oxygen-rich than thick disk stars as can be seen from Bensby et al. $(2004,2013)$ data.

Recently, based on APOGEE data (Majewski et al. 2016), bulge giants were found to be more Mg-rich than thick disk stars by Schultheis et al. (2017), relaunching the discussion as to whether the bulge formation timescale has been faster than that of the thick disk.

Magnesium: Figs. 6 and 7 show published magnesium abundances from Gonzalez et al. (2011), Johnson et al. (2014) and Alves-Brito et al. (2010) that revise the same stars as in Meléndez et al. (2008) and Ryde et al. (2016) for red giants, from
Hill et al. (2011) and Gonzalez et al. (2015) for Red Clump (RC) stars and from Bensby et al. (2013) for microlensed dwarfs. The data are compared with models with $v_{\mathrm{SF}}=3,1$ and $0.5 \mathrm{Gyr}^{-1}$.

In Figs. 6a and 7a,b, the models fit the Hill et al. (2011) data well, but underestimate the other published results.

Similarly to oxygen, magnesium is mainly produced during the hydrostatic nuclear burning phases of SNII progenitors (WW95; Kobayashi et al. 2006). However, even for the case of an archetypical $\alpha$ element such as magnesium, the nucleosynthesis prescription should take into account the incompleteness of the reaction network and uncertainties in the nuclear physics and the locus where the element is produced. In particular, Woosley and Weaver (1995) models underestimate the $\left[{ }^{25} \mathrm{Mg} /{ }^{24} \mathrm{Mg}\right]$ and $\left[{ }^{26} \mathrm{Mg} /{ }^{24} \mathrm{Mg}\right]$ ratios at low metallicities (Timmes et al. 1995) and in addition, most of the ${ }^{24} \mathrm{Mg}$ is not 


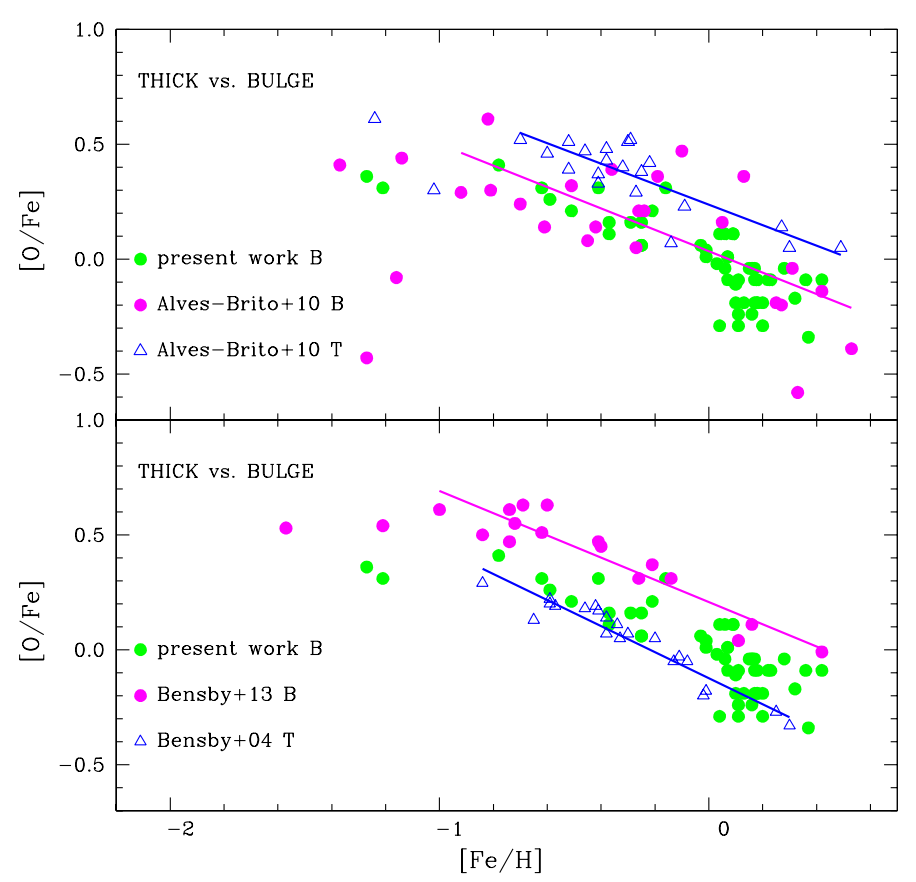

Fig. 5. $[\mathrm{O} / \mathrm{Fe}]$ vs. $[\mathrm{Fe} / \mathrm{H}]$ : present results for bulge giants compared to: a) thick disk and bulge giants. The lines are the linear regressions to the data with $[\mathrm{Fe} / \mathrm{H}] \geq-1.0$; the upper blue line is the linear regression to the thick disk data and the lower magenta line is the linear regression to the bulge data. by Alves-Brito et al. (2010); b) thick disk and bulge dwarfs by Bensby et al. (2004, 2013). The upper magenta line is the linear regression to the bulge data and the lower blue line is the linear regression to the thick disk data.

produced by the sequence of addition of $\alpha$ particles to ${ }^{12} \mathrm{C},{ }^{16} \mathrm{O}$ ${ }^{20} \mathrm{Ne}-\mathrm{Mg}^{24}$, but by direct carbon burning. Therefore, it is not recommended that other model parameters such as the IMF index be deduced from the $\mathrm{Mg}$ level of abundances. The underestimation of Mg yields by WW95 was already discussed by Argast et al. (2002), as being due to uncertainties in progenitor models prior to core-collapse, the crucial reaction rate ${ }^{12} \mathrm{C}(\alpha, \gamma){ }^{16} \mathrm{O}$, convection treatment and explosion mechanisms and energies. The uncertainties on these processes can been narrowed by new nuclear data and ab initio calculations. Accordingly, since WW95, there have been updates in the nucleosynthesis models of massive stars (Kobayashi et al. 2006; Nomoto et al. 2013; West et al. 2016). Recently, Sukhbold et al. (2016) presented yields with models where the explosion energies were predicted rather than assumed. According to the authors, the yields on $\mathrm{Mg}$ and $\mathrm{Al}$ are still slightly deficient, possibly due to a low CO core-size, which in turn may be a consequence of neglecting rotation or differences in overshooting mixing.

In Fig. $6 \mathrm{~b}$, the $[\mathrm{O} / \mathrm{Mg}]$ ratio, as extensively discussed by McWilliam (2016), shows a drop in $[\mathrm{O} / \mathrm{Mg}]$ for super-metalrich stars, which is not expected and could be due to selective winds in massive stars, as proposed by McWilliam (2016, and references therein), and regarded as a possibility by Kobayashi \& Nakasato (2011), with the other possibility being some process that would change the $\mathrm{C} / \mathrm{O}$ ratio. The present models include metallicity-dependent yields from stellar winds but do not discriminate among different elements.

Silicon, calcium, and titanium: Figs. 8-10 show the Si, Ca and $\mathrm{Ti}$ abundances in red giants published by Alves-Brito et al. (2010), Gonzalez et al. (2011), Johnson et al. (2014), and Ryde et al. (2016), in the Red Clump (RC) stars published by Gonza- lez et al. (2015), and in microlensed dwarfs published by Bensby et al. (2013). These figures show that the chemo-dynamical models for $v_{\mathrm{SF}}=0.5 \mathrm{Gyr}^{-1}$ fit the shape and location of the turnover, due to $\mathrm{Fe}$ enrichment by $\mathrm{SNIa}$, for $\mathrm{Si}$, and $\mathrm{Ca}$, remarkably well. The level of abundances is better reproduced with $v_{\mathrm{SF}}=3 \mathrm{Gyr}^{-1}$, but our results are sensitive to details of prescriptions. For Ti there could be a small shift between the models and the observations for the moderate metallicity interval $-1.0<[\mathrm{Fe} / \mathrm{H}]<-0.4$. As pointed out by Timmes et al. (1995), Ti is underproduced in their models by at least a factor of two. Following this remark by Timmes et al. (1995), in our models we used yields for the Ti that are double those given in WW95. Even applying this correction, however, Ti abundances are still somewhat underpredicted, as noted above.

An important conclusion is that the SNIa enrichment, indicated by the knee where the $[\mathrm{Si}, \mathrm{Ca} / \mathrm{Fe}]$ ratios start to drop, located at $[\mathrm{Fe} / \mathrm{H}] \sim-0.6-0.7$, is suitably well-defined by the models.

A further important conclusion, reached by Johnson et al. (2013), Ryde et al. (2016) and McWilliam (2016), is that these alpha-elements show the same behaviour in all different parts of the Galactic bulge so far studied.

\section{Conclusions}

The present chemical evolution models describe a classical bulge, and they are robust enough to drive conclusions on the timescales for bulge formation as well as on chemical enrichment at moderately low metallicities. The following conclusions are drawn from the comparison of models with data:

a) The model predictions including hypernovae as compared with those without them, indicate that the nucleosynthesis prescriptions are not critical for the $[\mathrm{O} / \mathrm{Fe}]$ ratios. In other words, the trend of $[\mathrm{O} / \mathrm{Fe}]$ vs. $[\mathrm{Fe} / \mathrm{H}]$ is a very robust indicator of the process of bulge formation. It also applies to the other alpha-elements, however their nucleosynthetic production is more complex and includes contributions from SNIa for $\mathrm{Ca}$ and $\mathrm{Ti}$. In general, the inclusion of hypernovae is only important for $[\mathrm{Fe} / \mathrm{H}]<-2.0$.

b) Another result from the models that is confirmed by the data is the $[\mathrm{alpha} / \mathrm{Fe}] \sim+0.25 \pm 0.5$ values at the solar metallicity $[\mathrm{Fe} / \mathrm{H}]=0.0$ for $\mathrm{Si}, \mathrm{Ca}$ and Ti. The $[\mathrm{Si}, \mathrm{Ca} / \mathrm{Fe}] \sim+0.25$ and $[\mathrm{Ti} / \mathrm{Fe}] \sim+0.10$ at $[\mathrm{Fe} / \mathrm{H}]=0.0$ is a consequence of a fast SFR in the bulge. For Mg, a high value only corresponds to the innermost bulge regions in the models. There is a spread in the data, and the Mg abundances by Hill et al. (2011) that appear reliable include stars with $[\mathrm{Mg} / \mathrm{Fe}] \sim 0.0$ at $[\mathrm{Fe} / \mathrm{H}]=0.0$, and these are well matched by the model predictions for the $0.5<r(\mathrm{kpc})<1$ region. However, as mentioned before, the nucleosynthesis prescriptions for $\mathrm{Mg}$ could be underestimates, as remarked by Timmes et al. (1995). For oxygen instead, $[\mathrm{O} / \mathrm{Fe}]$ is close to the solar value at $[\mathrm{Fe} / \mathrm{H}]=0.0$, which would imply a decrease in oxygen enrichment with increasing metallicity, as already pointed out by McWilliam et al. (2008) and McWilliam (2016).

c) The spread in the data is partially due to observational uncertainties, but on the other hand, a true spread is expected from the location of star formation, as can be seen in Figs. 3 and 4, where the model predictions are shown for shells at different distances from the Galactic centre. The intrinsic spread in the $[\mathrm{O} / \mathrm{Fe}]$ values reflects a spread in the radial distance at which star formation occurs. 
A. C. S. Friaça and B. Barbuy: Chemodynamical evolution model of alpha-elements in the Galactic bulge
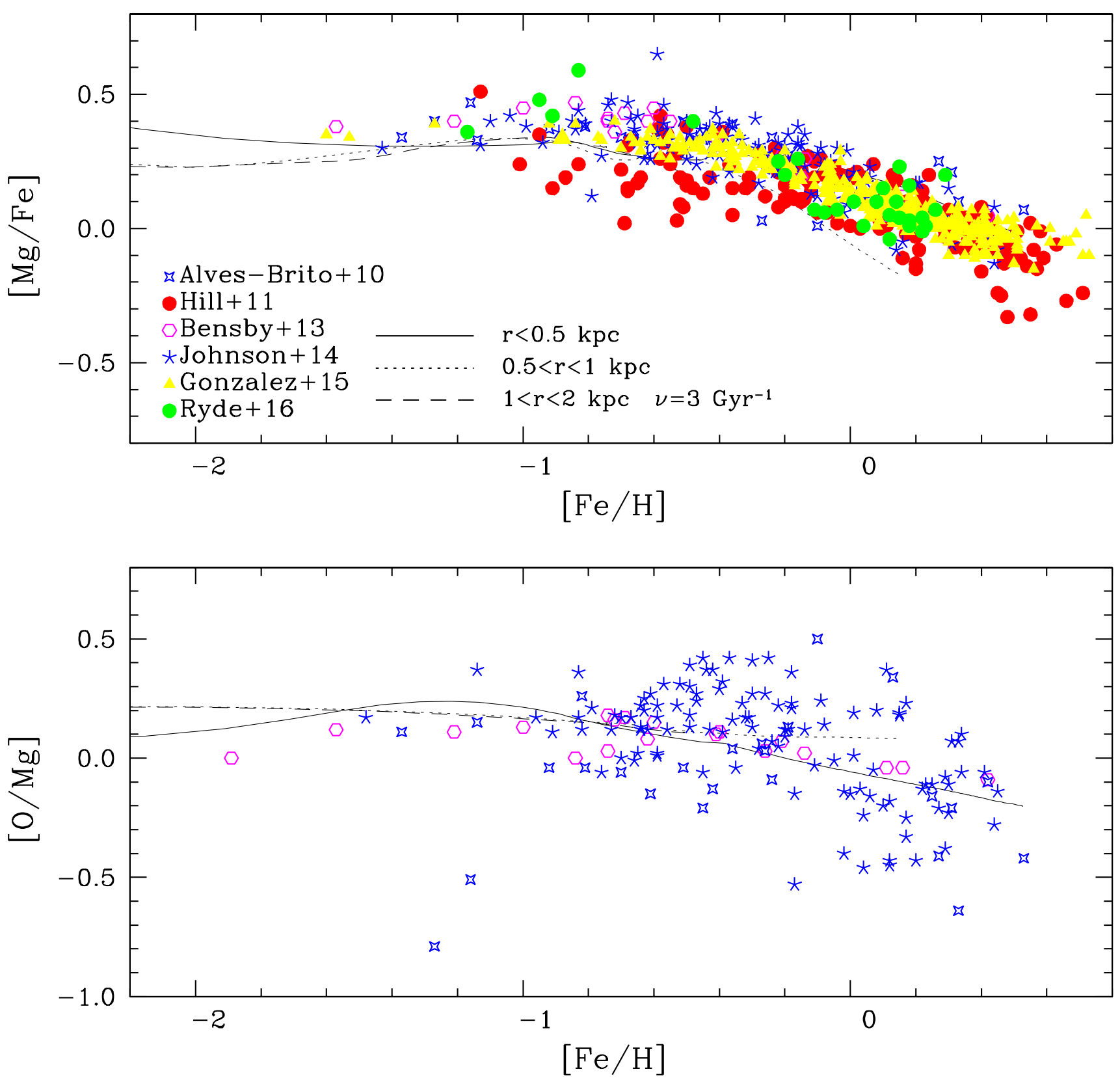

Fig. 6. $[\mathrm{Mg} / \mathrm{Fe}]$ vs. $[\mathrm{Fe} / \mathrm{H}]$ and $[\mathrm{O} / \mathrm{Mg}]$ vs. $[\mathrm{Fe} / \mathrm{H}]$ data from the literature for thick disk and bulge stars are compared to the predictions of the model with a specific star-formation rate of $v_{\mathrm{SF}}=3 \mathrm{Gyr}^{-1}$. The references for the data points are: Alves-Brito et al. (2010), Hill et al. (2011), Bensby et al. (2013), Johnson et al. (2014), Gonzalez et al. (2015) and Ryde et al. (2016).

d) As regards the IMF, as stated in Sect. 2, our adopted IMF assumes the usual Salpeter index $x_{\mathrm{IMF}}=1.35$ and extends to $100 M_{\odot}$, whereas some chemical evolution models by other authors assume a different IMF.

The possibility that the high mass cutoff could be smaller than the value of $100 M_{\odot}$, as explored by Tsujimoto et al. (1995), seems to be excluded by the statistics of gravitational wave sources, and in particular by the event GW150914 due to the merger of two $\sim 30 M_{\odot}$ black holes (Abbott et al. 2016). High-mass black holes shoud not be very rare, which implies that the IMF extends up to at least $100 M_{\odot}$ and probably up to $150 M_{\odot}$ (Belczynski et al. 2016), and even up to $300 M_{\odot}$ (Eldridge \& Stanway 2016). On the other hand, we tested the hypothesis of a top-heavy IMF (Tsujimoto \& Bekki 2012), by adopting their choice $x_{\mathrm{IMF}}=1.05$ for the model shown in
Fig. 4. It is clear that such models give oxygen abundances at all metallicities that are too high.

e) An important indicator of chemical enrichment suitability is the start of contributions by SNe Ia that appear as a turnover in the $[\alpha / \mathrm{Fe}]$ ratio, corresponding to the enrichment of Fe by SNe Ia.

The turnover point for the thick disk stars is seen at $-0.8<$ $[\mathrm{Fe} / \mathrm{H}]<-0.7$ in data points by Reddy et al. (2006), at $-0.7<[\mathrm{Fe} / \mathrm{H}]<-0.6$ in Alves-Brito et al. (2010), and at $[\mathrm{Fe} / \mathrm{H}] \sim-0.6$ in Bensby et al. (2004; see Fig. 3c).

For the bulge stars, the metallicity at which the turnover occurs is less clear from the data, given the spread in abundances: according to the present results, from oxygen (Figs. 2-4) it is approximately $-0.6<[\mathrm{Fe} / \mathrm{H}]<-0.7$, but there are not enough stars around these metallicities; it seems 

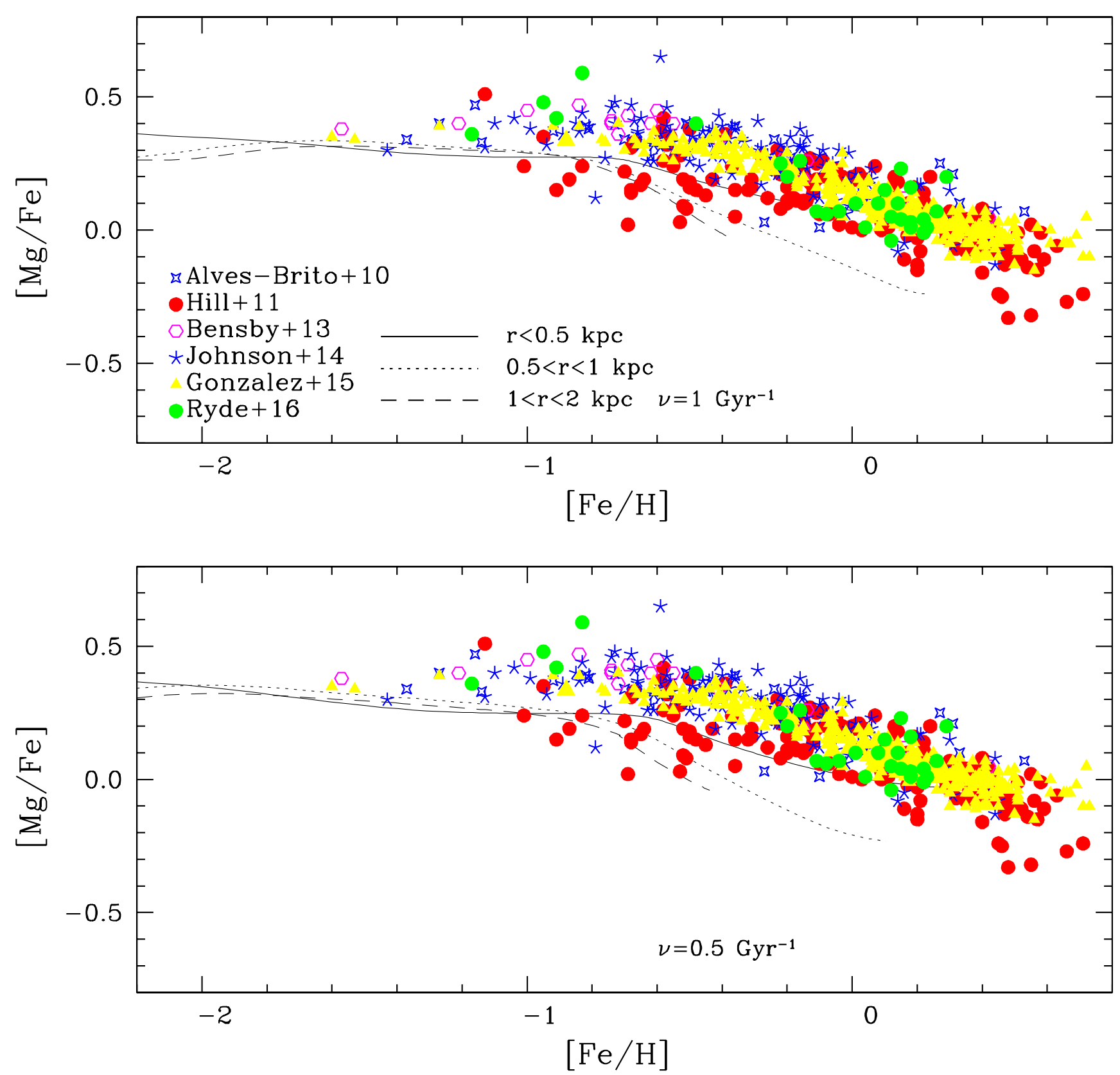

Fig. 7. $[\mathrm{Mg} / \mathrm{Fe}]$ vs. $[\mathrm{Fe} / \mathrm{H}]$ data from literature for thick disk and bulge stars are compared to the predicted $[\mathrm{Mg} / \mathrm{Fe}]$ vs. $[\mathrm{Fe} / \mathrm{H}]$ of the models with $v_{\mathrm{SF}}=1 \mathrm{Gyr}^{-1}$ and $v_{\mathrm{SF}}=0.5 \mathrm{Gyr}^{-1}$ using the references as in Fig. 6 .

to be at $[\mathrm{Fe} / \mathrm{H}] \sim-0.3$ from data provided by Johnson et al. (2014) and at $-0.6<[\mathrm{Fe} / \mathrm{H}]<-0.5$ from that provided by Bensby et al. (2013) and Ryde et al. (2010). The data on $[\mathrm{Mg} / \mathrm{Fe}]$ (Fig. 6a) indicate a knee at approximately $-0.8<$ $[\mathrm{Fe} / \mathrm{H}]<-0.6$. We note that the ratio $[\mathrm{Mg} / \mathrm{O}]$ should not be affected by yields from SNe Ia (Fig. 6b). [Si/Fe] and $[\mathrm{Ca} / \mathrm{Fe}]$ in Figs. 8 and 9 show a turnover at $-0.8<[\mathrm{Fe} / \mathrm{H}]<-0.6$. In the case of the $[\mathrm{Si} / \mathrm{Fe}]$ and $[\mathrm{Ca} / \mathrm{Fe}]$ trends with metallicity, the decrease is flatter in the range $-0.6<[\mathrm{Fe} / \mathrm{H}]<-0.2$ relative to $[\mathrm{Mg} / \mathrm{Fe}]$. As regards $\mathrm{Ti}$, there is no evidence of a knee from either models or observations (see Fig. 10).

f) The turnover is related to the question of how fast the bulge chemical enrichment was. The models for $v_{\mathrm{SF}}=0.5$ to $1.0 \mathrm{Gyr}^{-1}$ appear to be more suitable for producing the decrease in oxygen abundances at the higher metallicities, and also the oxygen abundances at the lower metallicities. The two data points at $[\mathrm{Fe} / \mathrm{H}] \sim-1.2$ would indicate the slower star-formation timescale of $2 \mathrm{Gyr}$.

On the other hand, the present models with $0.3 \mathrm{Gyr}^{-1}$ reproduce the higher oxygen abundances by Bensby et al. (2013) and Johnson et al. (2014) well. If the lower abundance points are correct, as we claim, they could provide information on (i) the nucleosynthetic process in low metallicity high-mass-type SNe II; (ii) the upper mass limit in the IMF (e.g. Tsujimoto et al. (1995) and (iii) the timescales of bulge formation.

The general trends of $\mathrm{Si}$ and $\mathrm{Ca}$ abundance ratios vs. $[\mathrm{Fe} / \mathrm{H}]$ are better fitted for $3 \mathrm{Gyr}^{-1}$ in terms of absolute abundances, whereas $0.5 \mathrm{Gyr}^{-1}$ better reproduces the turnover in $\mathrm{Si}$ and $\mathrm{Ca}$. For $\mathrm{Ti}$, the level of abundances tends to be lower in the models relative to the data. According to Timmes et al. (1995), their models underpredict the Ti abundances over all 
A. C. S. Friaça and B. Barbuy: Chemodynamical evolution model of alpha-elements in the Galactic bulge

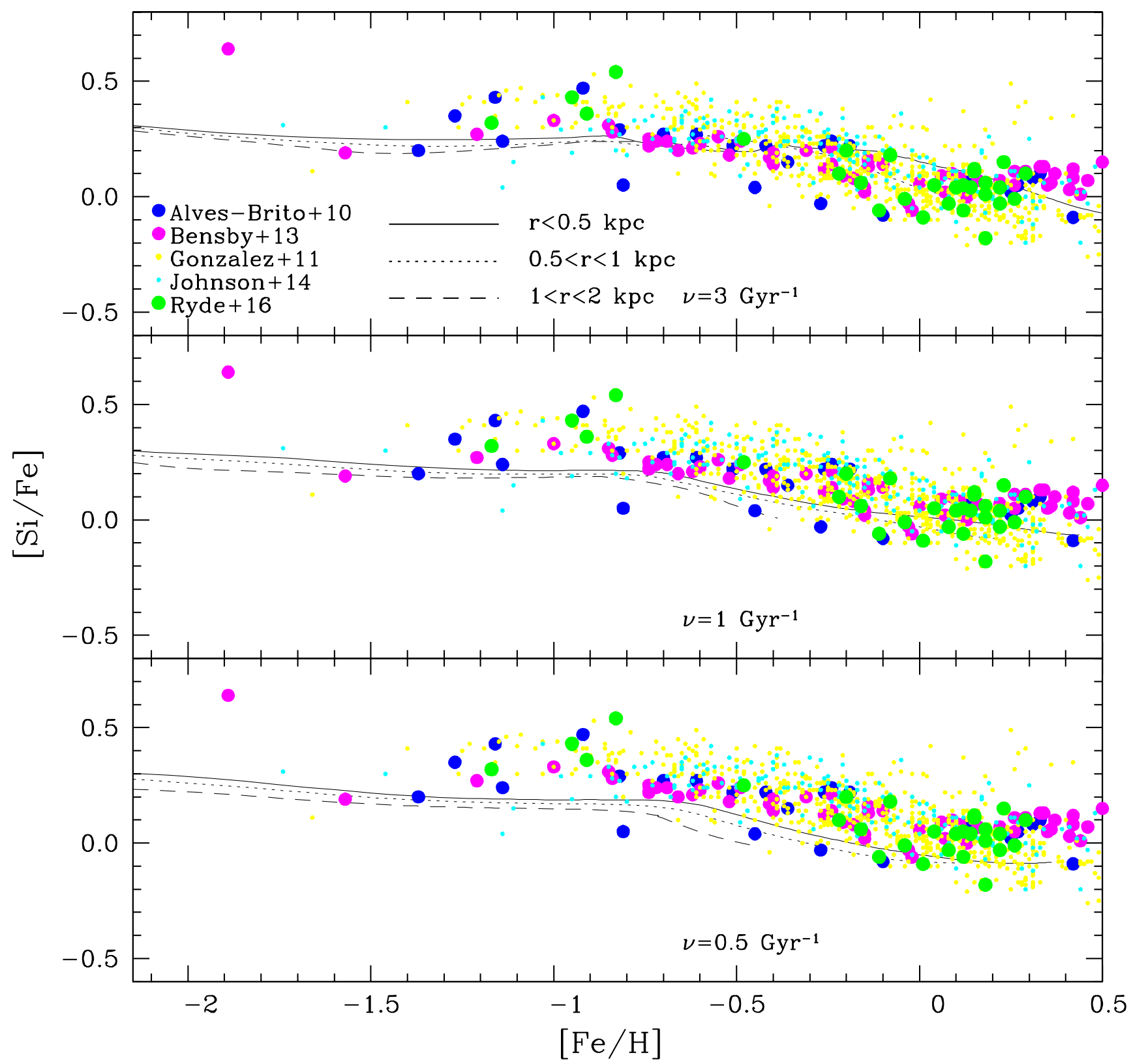

Fig. 8. $[\mathrm{Si} / \mathrm{Fe}]$ vs. $[\mathrm{Fe} / \mathrm{H}]$ data from the literature for halo and bulge stars are compared to the predictions of models with $v_{\mathrm{SF}}=3,1$ and $0.5 \mathrm{Gyr}{ }^{-1}$ using the same references as in Fig. 6.

metallicities. Therefore, the level of $\mathrm{Si}, \mathrm{Ca}$ and $\mathrm{Ti}$ abundances could be used to refine the nucleosynthesis prescriptions adopted in the model, in addition to being interpreted in terms of history of bulge formation. These elements result from both hydrostatic and explosive nucleosynthesis, which makes their predictions more complex than for $\mathrm{O}$ and $\mathrm{Mg}$ that are produced in hydrostatic phases only.

Kobayashi \& Nakasato (2011) presented a chemodynamical model for the Milky Way Galaxy. Their bulge component seems to be larger than the Galactic bulge, and should correspond better to the M31 bulge; in general, their calculations are applicable to the Galactic bulge. In Kobayashi \& Nakasato (2011; their Fig. 17), there is a transition at $[\mathrm{Fe} / \mathrm{H}] \sim-0.1$ to +0.3 that corresponds to a fast SFR. In their model, an initial starburst is induced by the assembling of proto-clumps, when most stars are formed in the first 2 Gyr.
Similarly, Nakasato \& Nomoto (2003), using a smoothed particle hydrodynamics (SPH) code for the evolution of the Galaxy with star-formation processes, found that a spheroidal bulge is formed early, and a bar is formed afterwards. They propose that the bulge has two components, an early subgalactic clump merger in the proto-Galaxy, and the bar that has formed gradually in the inner disk. At the stage of bulge formation from early mergers, $60 \%$ of bulge stars are formed in $0.5 \mathrm{Gyr}$. The turnover occurs at $[\mathrm{Fe} / \mathrm{H}] \sim 0$. The models by Cescutti \& Matteucci (2011), show a turnover at $[\mathrm{Fe} / \mathrm{H}] \sim$ +0.3 , due to a very fast timescale of $v_{\mathrm{SF}} \sim 10 \mathrm{Gyr}^{-1}$. A turnover at high metallicities of $[\mathrm{Fe} / \mathrm{H}] \sim 0.0$ is not confirmed by the data, and we suggest that the longer bulge formation timescales are more suitable.

g) A comparison with models including the formation of a bar was also carried out. Cavichia et al. (2014) models consider 


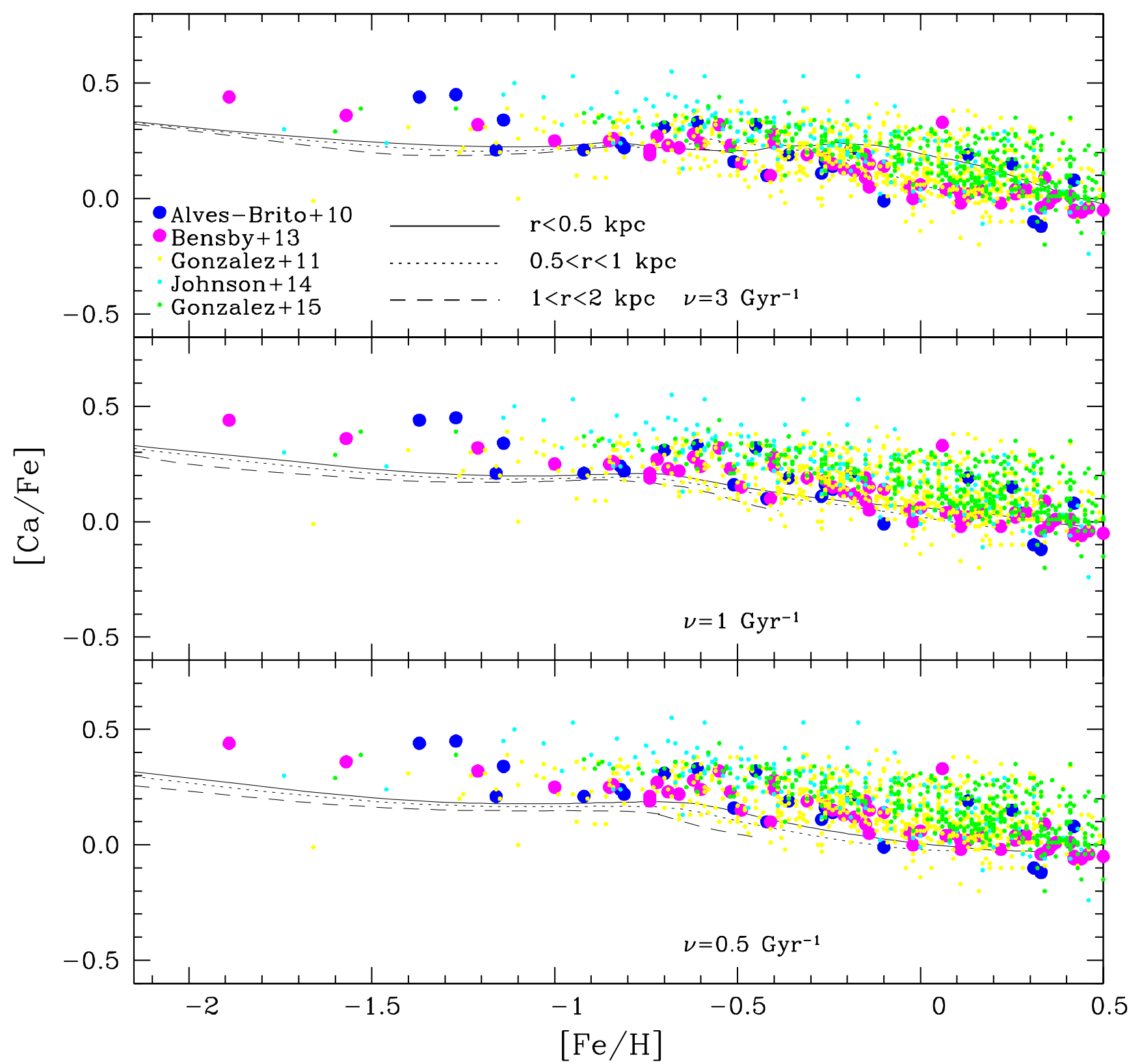

Fig. 9. $[\mathrm{Ca} / \mathrm{Fe}]$ vs. $[\mathrm{Fe} / \mathrm{H}]$ data from the literature for halo and bulge stars are compared to the predictions of models with $v_{\mathrm{SF}}=3,1$ and $0.5 \mathrm{Gyr}{ }^{-1}$ using the same references as in Fig. 6.

a bulge formed in a collapse timescale of $2 \mathrm{Gyr}$, or $v_{\mathrm{SF}}=$ $0.5 \mathrm{Gyr}^{-1}$. The inclusion of radial inflow of gas, through the bar, does not affect the bulge metallicity distribution function (MDF), given that the bulk of bulge stars formed in a much shorter timescale before bar formation. The bulge collapse timescale of 1-2 Gyr of Cavichia et al. (2014), similarly to our models with $v_{\mathrm{SF}}=1-0.5 \mathrm{Gyr}^{-1}$, better explain the data points with higher metallicity and lower $[\mathrm{O} / \mathrm{Fe}] \mathrm{ra}-$ tios (Fig. 4). For $\mathrm{Mg}$, the $v_{\mathrm{SF}}=0.5 \mathrm{Gyr}^{-1}$ fits the $\mathrm{Mg}$ data by Hill et al. (2011) very well, whereas even our higher $v_{\mathrm{SF}}=3 \mathrm{Gyr}^{-1}$ (fastest timescale) cannot fit the other literature data. McWilliam (2016) discusses the difficulties in deriving reliable $\mathrm{Mg}$ abundances.

Tsujimoto \& Bekki (2012) produce a fast formation of a metal-poor population in the bulge and thick disk, reaching $([\mathrm{Fe} / \mathrm{H}],[\mathrm{Mg} / \mathrm{H}],[\mathrm{Ba} / \mathrm{H}])=(-1.3,-0.9,-1.1)$; the metal-rich population forms subsequently from remaining gas, plus inflow from the inner disk, when the bar also forms (after the metalpoor population was formed). According to Nakasato \& Nomoto (2003), the bar forms after the bulge, therefore even if there is radial inflow of gas and stars into the bulge, this could be considered a late event in the evolution of a classical bulge. This two-stage formation history of the bulge is confirmed by the observations, given that the bulge shows the characteristics of a classical bulge and also that of a pseudo-bulge. Babusiaux $(2010,2014)$ has shown, from kinematics and metallicity data for a large sample of stars, that metal-poor stars are found in a spheroidal distribution, and metal-rich ones in a bar-like distribution.

Acknowledgements. We are very grateful for the extremely useful comments from the referee. A. C. S. F. and B. B. acknowledge partial financial support by $\mathrm{CNPq}, \mathrm{CAPES}$, and FAPESP. 
A. C. S. Friaça and B. Barbuy: Chemodynamical evolution model of alpha-elements in the Galactic bulge

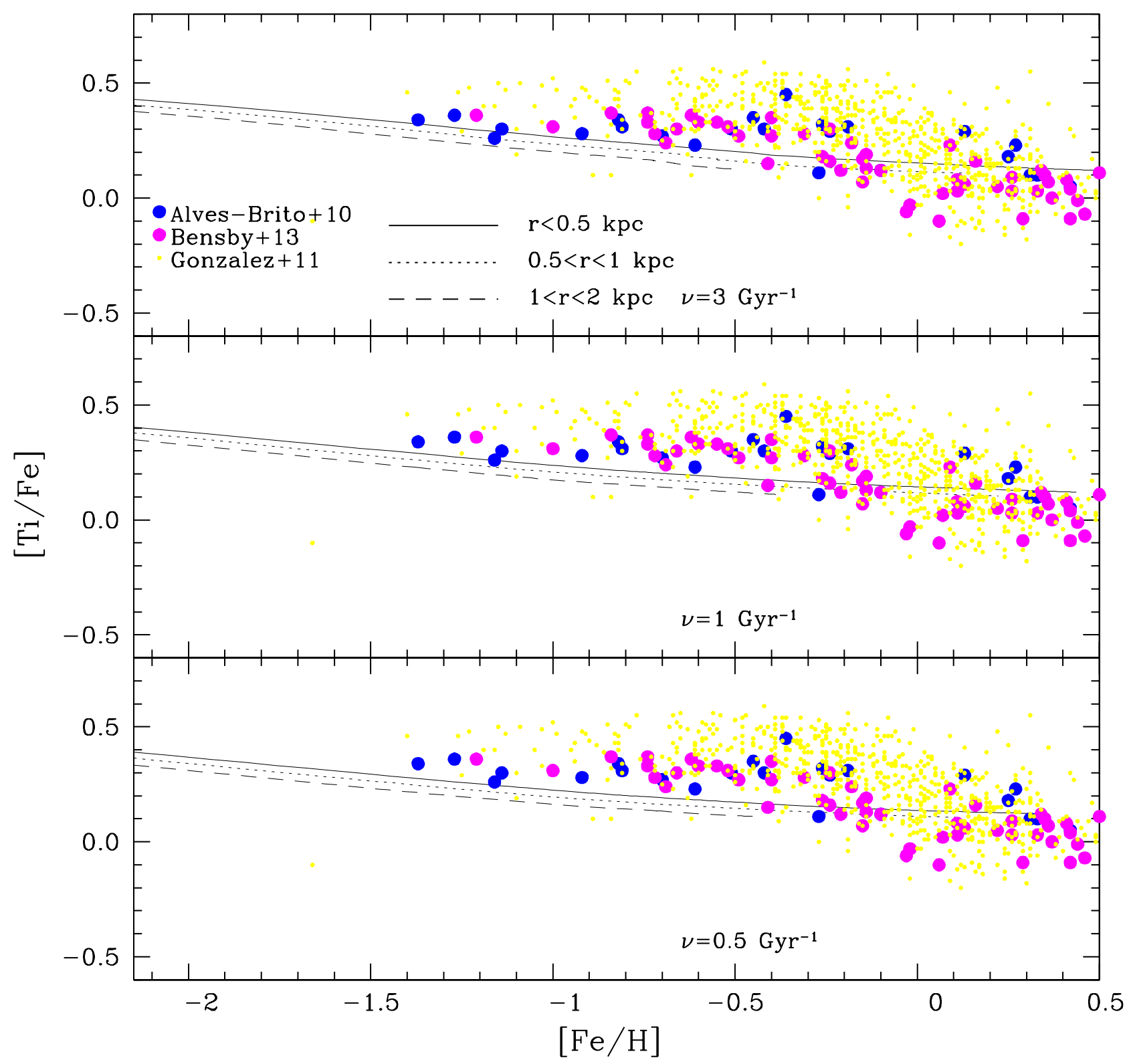

Fig. 10. $[\mathrm{Ti} / \mathrm{Fe}]$ vs. $[\mathrm{Fe} / \mathrm{H}]$ data from literature for halo and bulge stars are compared to the predictions of models with $v_{\mathrm{SF}}=3,1$ and $0.5 \mathrm{Gyr}{ }^{-1}$ using the same references as in Fig. 6.

\section{References}

Abbott, B. P., Abbott, R., Abbott, T. D., et al. 2016, Phys. Rev. Lett., 116, 061102 Allende Prieto, C., Lambert, D. L., \& Asplund, M. 2001, ApJ, 556, L63 Alves-Brito, A., Meléndez, J., Asplund, M., et al. 2010, A\&A, 513, A35

Argast, D., Samland, M., Thielemann, F.-K., \& Gerhard, O. E. 2002, A\&A, 388, 842

Asplund, M., Grevesse, N., Sauval, A. J., \& Scott, P. 2009, ARA\&A, 47, 481

Babusiaux, C., Gómez, A., Hill, V., et al. 2010, A\&A, 519, A77

Babusiaux, C., Katz, D., Hill, V., et al. 2014, A\&A, 563, A15

Ballero, S. K., Matteucci, F., Origlia, L., \& Rich, R. M. 2007, A\&A, 467, 123

Barbuy, B., Perrin, M.-N., Katz, D., et al. 2003, A\&A, 404, 661

Barbuy, B., Hill, V., Zoccali, M., et al. 2013, A\&A, 559, A5

Barbuy, B., Friaça, A., da Silveira, C. R., et al. 2015, A\&A, 580, A40

Belczynski, K., Holz, D. E., Bulik, T., \& O’Shaughnessy, R. 2016, Nature, 534, 512

Bensby, T., Feltzing, S., \& Lundström, I. 2004, A\&A, 415, 155

Bensby, T., Yee, J. C., Feltzing, S., et al. 2013, A\&A, 549, A147

Bensby, T., Feltzing, S., \& Oey, M. S. 2014, A\&A, 562, A71

Cardelli, J. A., Clayton, G. C., \& Mathis, J. S. 1989, ApJ, 345, 245

Cavichia, O., Mollá, M., Costa, R. D. D., \& Maciel, W. J. 2014, MNRAS, 437, 3688
Cescutti, G. \& Matteucci, F. 2011, A\&A, 525, A126

Coelho, P., Barbuy, B., Meléndez, J., et al. 2005, A\&A, 443, 735

Cunha, K., \& Smith, V. V. 2006, ApJ, 652, 491

Davis, S. P., \& Phillips, J. G. 1963 , The Red System $\left(\mathrm{A}^{2} \Pi-\mathrm{X}^{2} \Sigma\right)$ of the CN molecule (Berkeley: University of California Press)

Dobrovolskas, V., Kucinskas, A., Bonifacio, P., et al. 2015, A\&A, 576, A128

Eldridge, J. J., \& Stanway, E. R. 2016, MNRAS, 462, 3302

Friaça A. C. S., \& Terlevich R. J. 1998, MNRAS, 298, 399

Fröhlich, C., Hauser, P., Liebendörfer, M., et al. 2006, ApJ, 637, 415

Fulbright, J. P., McWilliam, A., \& Rich, R. M. 2006, ApJ, 636, 821

Fulbright, J. P., McWilliam, A., \& Rich, R. M. 2007, ApJ, 661, 1152

Gonzalez, O. A., Rejkuba, M., Zoccali, M., et al. 2011, A\&A, 530, A54

Gonzalez, O. A., Zoccali, M., Vasquez, S., et al. 2015, A\&A, 584, A46

Greggio, L., \& Renzini, A. 1983, A\&A, 118, 217

Grevesse, N., \& Sauval, J. N. 1998, Space Sci. Rev., 35, 161

Grieco, V., Matteucci, F., Pipino, A., \& Cescutti, G. 2012, A\&A, 548, A60

Gustafsson, B., Edvardsson, B., Eriksson, K., et al. 2008, A\&A, 486, 951

Hill, V., Lecureur, A., Gómez, A., et al. 2011, A\&A, 534, A80

Iwamoto, K., Brachwitz, F., Nomoto, K., et al. 1999, ApJS, 125, 439

Johnson, C. I., Rich, R. M., Kobayashi, C., et al. 2013, ApJ, 765, 157

Johnson, C. I., Rich, R. M., Kobayashi, C., et al. 2014, AJ, 148, 67

Kiselman, D. 2001, New Astron. Rev., 45, 559 
Kobayashi, C., \& Nakasato, N. 2011, ApJ, 729, 16

Kobayashi, C., Umeda, H., Nomoto, K., et al. 2006, ApJ, 653, 1145

Kurúcz, R. 1993, Kurucz CD-ROM No. 23 (Cambridge, Mass.: Smithsonian Astrophysical Observatory)

Lecureur, A., Hill, V., Zoccali, M., Barbuy, B., et al. 2007, A\&A, 465, 799

Lodders, K., Palme, H., Gail, H.-P. 2009, Landolt-Börnstein - Group VI Astronomy and Astrophysics Numerical Data and Functional Relationships in Science and Technology Volume 4B: Solar System, ed. J. E. Trümper, 4.4., 44

Majewski, S. R., Schiavon, R. P., Frinchaboy, P. M., et al. 2016, AJ, in press [arXiv: 1509.05420]

Matteucci, F. 1992, ApJ, 397, 32

Matteucci, F., \& Brocato, E. 1990, ApJ, 365, 539

Matteucci, F., \& Tornambè, A. 1987, A\&A, 185, 51

McWilliam, A. 2016, PASA, 33, 40

McWilliam, A., Matteucci, F., Ballero, S., et al. 2008, ApJ, 136, 367

Meléndez, J., Asplund, M., Alves-Brito, A., et al. 2008, A\&A, 484, L21

Nakasato, N., \& Nomoto, K. 2003, ApJ, 588, 842

Nissen, P. E., Primas, F., Asplund, M., \& Lambert, D. L. 2002, A\&A, 390, 235

Nomoto, K., Tominaga, N., Umeda, H., Kobayashi, C., \& Maeda, K. 2006, Nucl Phys. A, 777, 424

Nomoto, K., Kobayashi, C., \& Tominaga, N. 2013, ARA\&A, 51, 457
Planck Collaboration I. 2016, A\&A, 594, A1

Reddy, B. E., Lambert, D. L., \& Allende Prieto, C. 2006, MNRAS, 367, 1329 Rich, R. M., \& Origlia, L. 2005, ApJ, 634, 1293

Rich, R. M., Origlia, L., \& Valenti, E. 2012, ApJ, 746, 59

Ryde, N., Gustafsson, B., Edvardsson, B., et al. 2010, A\&A, 509, A20

Ryde, N., Schultheis, M., Grieco, V., et al. 2016, AJ, 151, 1

Samland, M., \& Gerhard, O. E. 2003, A\&A, 399, 961

Schultheis, M., Rojas-Arriagada, A., García-Perez, A. E., et al. 2017, A\&A, accepted

Steffen, M., Prakapavicius, D., Caffau, E., et al. 2015, A\&A, 583, A57

Sukhbold, T., Ertl, T., Woosley, S. E., et al. 2016, ApJ, 821, 38

Timmes, F. X., Woosley, S. E., \& Weaver, T. A. 1995, ApJS, 98, 617

Tsujimoto, T., \& Bekki, K. 2012, ApJ, 747, 125

Tsujimoto, T., Yoshii, Y., \& Nomoto, K., Shigeyama, T. 1995, A\&A, 302, 704 Umeda, H., \& Nomoto, K. 2002, ApJ, 565, 385

Umeda, H., \& Nomoto, K. 2003, Nature, 422, 871

Umeda, H., \& Nomoto, K. 2005, ApJ, 619, 427

West, C., Heger, A., \& Austin, S. M. 2013, ApJ, 769, 2

van de Hoek, L. B., \& Groenewegen M. A. T. 1997, A\&A, 123, 305

Woosley, S. E., \& Weaver, T. A. 1995, ApJS, 101, 181

Wyse, R. F. G., \& Gilmore, G. 1992, AJ, 104, 144

Zoccali, M., Lecureur, A., Barbuy, B., et al. 2006, A\&A, 457, L1

Zoccali, M., Hill, V., Lecureur, A., et al. 2008, A\&A, 486, 177 
A. C. S. Friaça and B. Barbuy: Chemodynamical evolution model of alpha-elements in the Galactic bulge

Appendix A: Re-derived C, N, and $\mathrm{O}$ abundances

Table A.1. Atmospheric parameters adopted from Zoccali et al. (2006), Lecureur et al. (2007) and Hill et al. (2011).

\begin{tabular}{|c|c|c|c|c|c|c|c|c|c|c|c|}
\hline Star & $T_{\text {eff }}$ & $\log g$ & $v_{\mathrm{t}}$ & {$[\mathrm{Fe} / \mathrm{H}]$} & {$[\mathrm{C} / \mathrm{Fe}]$} & {$[\mathrm{N} / \mathrm{Fe}]$} & {$[\mathrm{O} / \mathrm{Fe}]$} & {$[\mathrm{C}, \mathrm{N}, \mathrm{O} / \mathrm{Fe}]_{\text {corr }}$} & {$[\mathrm{C} / \mathrm{Fe}]$} & {$[\mathrm{N} / \mathrm{Fe}]$} & {$[\mathrm{O} / \mathrm{Fe}]$} \\
\hline B6-b1 & 4400 & 1.8 & 1.6 & 0.07 & -0.16 & 0.39 & 0.04 & $\mathrm{c}, \mathrm{c}, \mathrm{c}$ & -0.15 & +0.50 & 0.00 \\
\hline B6-b2 & 4200 & 1.5 & 1.4 & -0.01 & - & - & - & $-0.2,0.6,-0.1$ & -0.05 & +0.35 & 0.00 \\
\hline B6-b3 & 4700 & 2.0 & 1.6 & 0.10 & -0.16 & 0.11 & 0.19 & c, $0.3,-0.15$ & -0.25 & +0.50 & -0.12 \\
\hline B6-b4 & 4400 & 1.9 & 1.7 & -0.41 & -0.14 & 0.53 & 0.53 & $\mathrm{c}, \mathrm{c}, 0.3$ & -0.15 & +0.15 & +0.30 \\
\hline B6-b5 & 4600 & 1.9 & 1.8 & -0.37 & -0.11 & 0.56 & 0.33 & c, $0.3,0.15$ & -0.10 & +0.30 & +0.15 \\
\hline B6-b6 & 4600 & 1.9 & 1.8 & 0.11 & -0.03 & 0.57 & 0.01 & $-0.1,0.7,0.0$ & -0.15 & +0.50 & -0.10 \\
\hline B6-b8 & 4100 & 1.6 & 1.3 & 0.03 & 0.08 & 0.05 & 0.10 & $0, \mathrm{c}, 0$ & 0.00 & +0.10 & -0.03 \\
\hline B6-f1 & 4200 & 1.6 & 1.5 & -0.01 & 0.05 & 0.55 & 0.18 & $0.0,0.35,0.07$ & +0.05 & +0.20 & +0.03 \\
\hline B6-f2 & 4700 & 1.7 & 1.5 & -0.51 & -0.04 & 0.56 & 0.39 & $0,0.05,0.18$ & +0.0 & +0.20 & +0.20 \\
\hline B6-f3 & 4800 & 1.9 & 1.3 & -0.29 & -0.09 & 0.53 & 0.18 & c, $0.3,0.05$ & -0.05 & +0.30 & +0.15 \\
\hline B6-f5 & 4500 & 1.8 & 1.4 & -0.37 & 0.37 & 0.53 & - & $-0.1,0.15,0.0$ & +0.05 & +0.00 & +0.10 \\
\hline B6-f7 & 4300 & 1.7 & 1.6 & -0.42 & 0.42 & 0.57 & - & $0,0.3,0.1$ & 0.00 & +0.30 & - \\
\hline B6-f8 & 4900 & 1.8 & 1.6 & 0.04 & -0.11 & 0.51 & -0.17 & $\mathrm{c}, 0.35,-0.3$ & -0.10 & +0.30 & -0.20 \\
\hline BW-b2 & 4300 & 1.9 & 1.5 & 0.22 & 0.05 & 0.25 & 0.23 & $-0.1,0.1,-0.1$ & -0.10 & +0.20 & -0.10 \\
\hline BW-b4 & 4300 & 1.4 & 1.4 & 0.07 & - & - & - & $-0.1,0,-0.1$ & -0.10 & 0.00 & -0.10 \\
\hline BW-b5 & 4000 & 1.6 & 1.2 & 0.17 & 0.06 & 0.56 & 0.09 & $\mathrm{c}, 0,0$ & 0.00 & +0.05 & -0.10 \\
\hline BW-b6 & 4200 & 1.7 & 1.3 & -0.25 & 0.05 & 0.56 & 0.09 & $-0.3,0.9,0.25$ & 0.00 & +0.65 & +0.15 \\
\hline BW-b7 & 4200 & 1.4 & 1.2 & 0.10 & - & - & - & $-0.23,-0.1,-0.2$ & -0.25 & +0.10 & -0.20 \\
\hline BW-f1 & 4400 & 1.8 & 1.6 & 0.32 & -0.26 & 0.24 & -0.02 & $-0.2,0.5,-0.15$ & -0.20 & +0.45 & -0.18 \\
\hline BW-f4 & 4800 & 1.9 & 1.7 & -1.21 & 0.04 & 0.54 & - & c, c, 0.3 & +0.30 & +0.30 & +0.30 \\
\hline BW-f5 & 4800 & 1.9 & 1.3 & -0.59 & 0.03 & 0.53 & 0.31 & $\mathrm{c}, 0.2,0.1$ & +0.10 & +0.40 & +0.25 \\
\hline BW-f6 & 4100 & 1.7 & 1.5 & -0.21 & 0.08 & 0.58 & 0.46 & $\mathrm{c}, 0.4,0.18$ & +0.08 & +0.40 & +0.20 \\
\hline BW-f7 & 4400 & 1.9 & 1.7 & 0.11 & -0.10 & 0.30 & 0.03 & $-0.2,0.6,-0.1$ & -0.20 & +0.70 & -0.25 \\
\hline BW-f8 & 5000 & 2.2 & 1.8 & -1.27 & 0.03 & 0.53 & - & $0,+0.2,+0.5$ & +0.00 & +0.20 & +0.35 \\
\hline BL-1 & 4500 & 2.1 & 1.5 & -0.16 & 0.03 & 0.18 & 0.26 & $0.15,0.4,0.3$ & +0.15 & +0.40 & +0.30 \\
\hline BL-3 & 4500 & 2.3 & 1.4 & -0.03 & -0.07 & 0.18 & 0.22 & c, $0.3,0.1$ & +0.07 & 0.00 & +0.05 \\
\hline BL-4 & 4700 & 2.0 & 1.5 & 0.13 & -0.04 & 0.41 & 0.02 & $-0.1,0.3,-0.2$ & -0.10 & +0.20 & -0.20 \\
\hline BL-5 & 4500 & 2.1 & 1.6 & 0.16 & 0.04 & 0.33 & 0.07 & $-0.02, c,-0.05$ & +0.00 & +0.40 & -0.05 \\
\hline BL-7 & 4700 & 2.4 & 1.4 & -0.47 & -0.17 & 0.38 & 0.46 & $\mathrm{c}, \mathrm{c}, 0.3^{t}$ & +0.00 & +0.30 & +0.30 \\
\hline B3-b1 & 4300 & 1.7 & 1.5 & -0.78 & -0.10 & 0.45 & 0.55 & $0.1,0.6,0.4$ & $(0.00)$ & +0.60 & +0.35 \\
\hline B3-b2 & 4500 & 2.0 & 1.5 & 0.18 & -0.13 & 0.42 & 0.12 & c, $0,-0.1$ & -0.10 & +0.20 & -0.10 \\
\hline B3-b3 & 4400 & 2.0 & 1.5 & 0.18 & -0.09 & 0.46 & -0.19 & $0,0,-0.15$ & -0.10 & 0.00 & -0.20 \\
\hline B3-b4 & 4500 & 2.1 & 1.7 & 0.17 & -0.16 & 0.39 & -0.06 & $\mathrm{c}, \mathrm{c}, \mathrm{c}$ & -0.15 & +0.40 & -0.05 \\
\hline B3-b5 & 4600 & 2.0 & 1.5 & 0.11 & -0.15 & 0.40 & 0.00 & $-0.2,0,-0.3$ & -0.20 & 0.00 & -0.30 \\
\hline B3-b7 & 4400 & 1.9 & 1.3 & 0.20 & -0.16 & 0.39 & -0.06 & $\mathrm{c}, 0,-0.25$ & -0.15 & +0.25 & -0.20 \\
\hline B3-b8 & 4400 & 1.8 & 1.4 & -0.62 & -0.16 & 0.39 & 0.52 & c, $0.3,0.25$ & -0.15 & +0.15 & +0.30 \\
\hline B3-f1 & 4500 & 1.9 & 1.6 & 0.04 & 0.09 & 0.44 & 0.19 & $0,0.4,0.1$ & 0.00 & +0.40 & +0.10 \\
\hline B3-f3 & 4400 & 1.9 & 1.7 & 0.06 & -0.08 & 0.47 & -0.08 & $0,0, \mathrm{c}$ & +0.00 & +0.00 & -0.10 \\
\hline B3-f4 & 4400 & 2.1 & 1.5 & 0.09 & 0.10 & 0.45 & 0.43 & c, $0.1,0.1$ & 0.00 & +0.10 & +0.10 \\
\hline B3-f5 & 4200 & 2.0 & 1.8 & 0.16 & -0.06 & 0.49 & 0.09 & $\mathrm{c}, \mathrm{c},-0.05$ & -0.05 & +0.50 & -0.05 \\
\hline B3-f7 & 4800 & 2.1 & 1.7 & 0.16 & -0.02 & 0.33 & 0.05 & $\mathrm{c}, \mathrm{c}, \mathrm{c}$ & +0.00 & +0.20 & -0.25 \\
\hline B3-f8 & 4800 & 1.9 & 1.5 & 0.20 & -0.17 & 0.38 & 0.00 & c, $0.28,-0.25$ & -0.20 & +0.30 & -0.30 \\
\hline BWc-1 & 4476 & 2.1 & 1.5 & 0.09 & 0.12 & 0.50 & 0.19 & $0.05,0.3,0.1$ & +0.05 & +0.30 & +0.10 \\
\hline BWc-2 & 4558 & 2.2 & 1.2 & 0.18 & -0.09 & 0.52 & 0.07 & $-0.2,0.2,-0.15$ & -0.20 & +0.15 & -0.20 \\
\hline BWc-3 & 4513 & 2.1 & 1.3 & 0.28 & 0.04 & 0.51 & 0.19 & $-0.1,0.4,0$ & -0.10 & +0.40 & -0.05 \\
\hline BWc-4 & 4866 & 2.2 & 1.3 & 0.06 & 0.36 & 0.28 & 0.05 & $-0.1,0.05,-0.05$ & -0.10 & +0.05 & -0.05 \\
\hline BWc-5 & 4535 & 2.1 & 1.5 & 0.42 & -0.01 & 0.59 & 0.04 & $-0.05,0.4,-0.1$ & -0.05 & +0.30 & -0.10 \\
\hline BWc-6 & 4769 & 2.2 & 1.3 & -0.25 & -0.20 & 0.69 & 0.43 & $\mathrm{c}, \mathrm{c}, 0.25$ & -0.20 & +0.70 & +0.05 \\
\hline BWc-7 & 4590 & 2.2 & 1.1 & -0.25 & -0.20 & 0.50 & 0.44 & $\mathrm{c}, 0.3,0.25$ & -0.20 & +0.30 & +0.05 \\
\hline BWc-8 & 4610 & 2.2 & 1.3 & 0.37 & -0.22 & 0.47 & -0.07 & c, $0.1,-0.35$ & -0.30 & +0.10 & -0.35 \\
\hline BWc-9 & 4539 & 2.1 & 1.5 & 0.15 & -0.13 & 0.77 & 0.11 & $-0.1,0.2,-0.05$ & -0.10 & +0.20 & -0.05 \\
\hline BWc-10 & 4793 & 2.2 & 1.3 & 0.07 & -0.15 & 0.54 & 0.15 & $-0.2,0.3,0$ & -0.20 & +0.30 & 0.00 \\
\hline BWc-11 & 4576 & 2.1 & 1.0 & 0.17 & -0.14 & 0.56 & - & $-0.2,0,-0.2$ & -0.20 & 0.00 & -0.20 \\
\hline BWc-12 & 4547 & 2.1 & 1.3 & 0.23 & 0.19 & 0.29 & 0.24 & $-0.1,0.1,-0.05$ & -0.15 & +0.05 & -0.10 \\
\hline BWc-13 & 4584 & 2.1 & 1.1 & 0.36 & 0.12 & 0.51 & 0.13 & $0,-0.15,-0.1$ & 0.00 & -0.15 & -0.10 \\
\hline
\end{tabular}

Notes. CNO abundances are reported: Cols. 6-8 give Zoccali et al. (2006) and Lecureur et al. (2007) values, Col. 9 gives the values revised by Barbuy et al. (2015) and Cols. 10-12 give the present final values. 